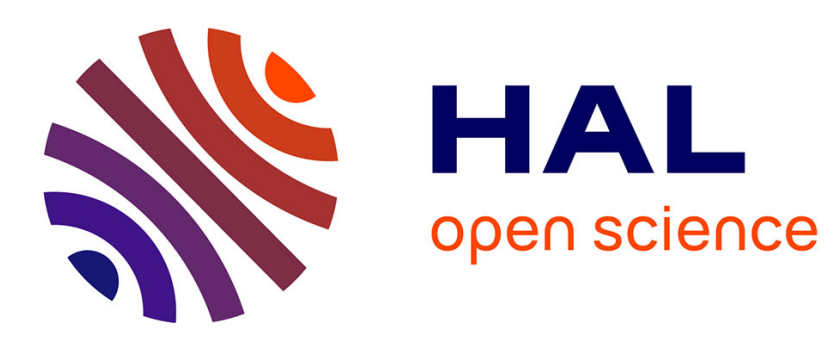

\title{
Random-phase approximation correlation methods for molecules and solids
}

\author{
Andreas Goerling, Andreas Hesselmann
}

\section{To cite this version:}

Andreas Goerling, Andreas Hesselmann. Random-phase approximation correlation methods for molecules and solids. Molecular Physics, 2011, pp.1. 10.1080/00268976.2011.614282 . hal-00741180

\author{
HAL Id: hal-00741180 \\ https://hal.science/hal-00741180
}

Submitted on 12 Oct 2012

HAL is a multi-disciplinary open access archive for the deposit and dissemination of scientific research documents, whether they are published or not. The documents may come from teaching and research institutions in France or abroad, or from public or private research centers.
L'archive ouverte pluridisciplinaire HAL, est destinée au dépôt et à la diffusion de documents scientifiques de niveau recherche, publiés ou non, émanant des établissements d'enseignement et de recherche français ou étrangers, des laboratoires publics ou privés. 


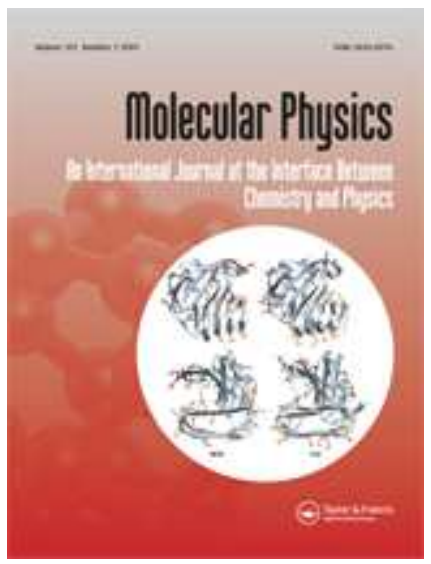

\section{Random-phase approximation correlation methods for molecules and solids}

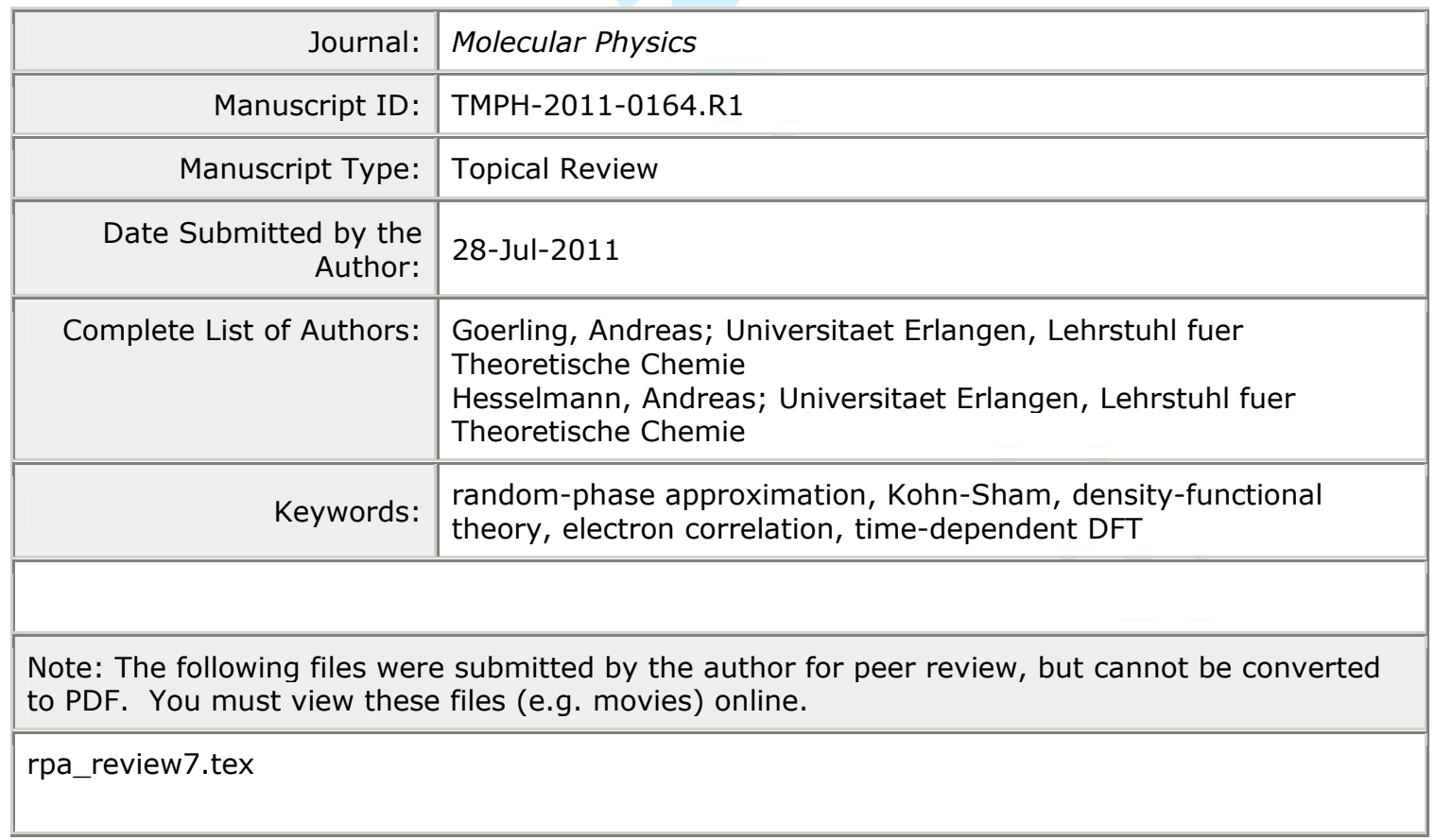

\section{SCHOLARONE Manuscripts}


Molecular Physics

Vol. 00, No. 00, Month 2009, 1-43

\title{
ARTICLE \\ Random-phase approximation correlation methods for molecules and solids
}

\author{
A. Heßelmann ${ }^{a *}$ and A. Görling ${ }^{a}$ \\ ${ }^{a}$ Lehrstuhl für Theoretische Chemie, Universität Erlangen-Nürnberg, Egerlandstr. 3, \\ D-91058 Erlangen, Germany; \\ (February 2011)
}

\begin{abstract}
Random-phase approximation (RPA) correlation methods based on Kohn-Sham densityfunctional theory and Hartree-Fock are derived using the adiabatic-connection fluctuation dissipation theorem. It is shown that the correlation energy within the adiabatic-connection fluctuation dissipation theorem is exact in a Kohn-Sham framework while for Hartree-Fock reference states this is not the case. This shows that Kohn-Sham reference states are probably better suited to describe electron correlation for use in RPA methods than Hartree-Fock reference states. Both, Kohn-Sham and Hartree-Fock RPA methods are related to each other both by comparing the underlying correlation functionals and numerically through the comparison of total energies and reaction energies for a set of small organic molecules.
\end{abstract}

Keywords: random-phase approximation, density functional theory, time-dependent density-functional theory, correlation energy, RPA

\section{Introduction}

In the early 1950's Bohm and Pines published a series of seminal papers that studied the collective properties of the electron gas [1-3]. They described the density fluctuations within the electron gas by separating a collective long-range (plasma) oscillation and a short-range thermal or random motion of the individual electrons. In their quantum mechanical treatment [3], the many-electron Hamiltonian was expressed through a Fourier transformation by a series of momentum transfers between the electrons and it was found that terms with a random phase, corresponding to different momentum transfers, have a zero mean value and can be neglected if the electrons interact rather weakly via screened Coulomb forces. Thus, within this random-phase approximation (RPA), the electrons are assumed to respond only to the total electric potential, which is the sum of an external potential and a screening potential produced by the electron clouds surrounding the electrons, as has been shown later on by Nozieres and Pines [4]. A relation between the RPA and the perturbed self-consistent field theory describing the interaction of a many-electron system with an electromagnetic field was then established by Ehrenreich and Cohen [5].

Using the plasmon model that describes the many-body system in terms of collective excitations, Sawada et al. derived an expression for the (exchange-)correlation energy that arises from the plasma oscillations which is given by the zero-point

*Corresponding author. Email: andreas.hesselmann@chemie.uni-erlangen.de 
energy of the oscillations and the value this energy approaches as the coupling between the oscillators is switched off [6]:

$$
E_{\mathrm{xc}}=\frac{1}{2} \sum_{i}\left[\omega_{i}-\omega_{i}^{0}\right]
$$

While this result differed from the corresponding expression of Bohm and Pines, since in their work the zero point energy alone appears explicitly [3], Sawada et al. showed that both approaches are identical in the high density limit. Thus the correlation energy within the random-phase approximation can also be connected with the zero-point energy of a set of harmonic oscillators which have the same oscillation frequencies as the electrons. This model is also closely related to a Drude model firstly introduced by London $[7,8]$ describing the dispersion interaction between atoms. In the Drude model the electron interactions are described by coupled harmonic oscillators such that, in a quantum mechanical picture, a zero-point energy exists describing a lowering of the energy due to correlations. Gell-Mann and Brueckner [9] have shown that the RPA correlation energy of an electron gas in the high density limit is represented by the sum of all Feynman diagrams with a ring structure and therefore the RPA also is sometimes refered to as ring approximation in many-particle physics.

Originally, electron exchange effects were neglected in early works using the RPA. This RPA method neglecting exchange effects shall be designated direct RPA (dRPA) here. However, it has been found later on, e.g., in the work of Brener and Fry, $[10,11]$ that an extension of the RPA dielectric function [12] using exchange interactions leads to improvements for the description of the properties of the electron gas. McLachlan and Ball derived an expression for the correlation energy of the electron gas within the framework of time-dependent Hartree-Fock (TDHF) theory $[13,14]$ that, however, differed from the original expression from Sawada et al. [6] by a factor of one half. While the TDHF approach itself has been used extensively for the description of excited states and dynamic response properties [15-20], the TDHF RPA variant from McLachlan and Ball has rarely been used for describing correlated molecular ground states in contrast to the original RPA method excluding exchange interactions. On the one hand, the reason for this may stem from the fact that the TDHF method often is affected by triplet instabilities due to an incompatibility of the wave function ansatz and the excitation operators $[14,21-25]$. On the other hand, it turned out that alternative correlation methods like many-body perturbation theory or coupled-cluster theory are more accurate for the description of the correlation energy of molecules.

The RPA approach for determining the correlation energy of a many-body system can be derived from a very general theorem, termed adiabatic-connection fluctuation-dissipation theorem (AC-FDT) [26-29] which apparently first was first discovered by Pauli (see remarks in Refs. $[4,26]$ ). The fluctuation-dissipation theorem was originally derived by Callen and Welton [30] and is used to study the properties of non-equilibrium thermodynamic systems. It relates the internal random motions of the particles in a many-body system to the response to small externally applied perturbations since both, the fluctuation forces and the dissipative forces (that is the density-density response) have their origin in the interactions between the particles. The AC-FDT theorem within the framework of the Kohn-Sham (KS) formalism of density-functional theory (DFT) [28, 29] provides an exact expression for the exchange- and correlation-energy, while an approximate expression for the exchange- and correlation-energy is provided on the basis of the HartreeFock (HF) method. The AC-FDT requires as input the response function of the 
electronic system. The latter has to be approximated. If this response function is determined via TDHF then TDHF RPA methods are obtained. If the response function is determined within time-dependent density-functional theory (TDDFT) then KS based RPA correlation energies result.

While RPA methods based on HF have rarely been used in molecular applications since their invention (see also a recent work by Klopper et al. which suggests that RPA variants including Hartree-Fock exchange seem to be less suitable to describe electron ground-state correlation than direct RPA methods [31]), see above, in recent years Kohn-Sham based RPA methods have gained an increase in popularity [32-55]. Apart from presenting efficient computational implementations of direct RPA methods for solids and molecules [39, 40, 45], it was shown that direct RPA methods using KS orbitals yield quite accurate results for atomisation energies and even describe to some extent static correlation effects in molecules [33]. This finding is somewhat surprising in view of the fact that direct RPA methods violate the Pauli exclusion principle since they treat on an unequal footing so called exclusionprinciple violating diagrams, i.e., diagrams of equal magnitude and opposite sign that cancel each other in a complete perturbation series expansion. Because of this it has been argued [56] that direct RPA methods may fail especially for small systems and small basis sets while they should produce better results for extended systems, e.g., the electron gas. Indeed the direct RPA exhibits the shortcoming of yielding a nonzero correlation energy in one-electron systems while it has been demonstrated recently that direct RPA calculations of a set of 24 solids yield excellent lattice constants and good relative energies [46, 57].

In spite of recent developments that make RPA methods more efficient for molecular systems, they are still computationally more demanding than standard KohnSham DFT methods. This stems from the fact that within the RPA one has to accurately model the interelectronic cusp, a problem that is common in all orbital based correlation methods. Usually this requires large basis sets with high angluar momentum functions. A potential remedy of this problem are range-separated methods $[42,43,49,50,58-63]$ in which the short-range electron correlation is treated via conventional DFT methods and the long-range correlation via orbital based methods. While such methods originally treated the long-range part or the correlation with configuration-interaction [58] or second-order Møller-Plesset [59] methods, it was found in a number of recent studies that range-separated methods including long-range RPA (including or excluding exchange) perform well for a number of molecular properties [42, 43, 49, 50, 62, 63].

The TDDFT methods used in KS based RPA approaches to calculate the response function require approximations for the exchange-correlation potential and kernel (more precisely, for the exchange potential and kernel, while KS methods that include electron correlation effects in the potential and kernel go beyond the random-phase approximation, see, e.g., Ref. [64]). The exchange-correlation kernel is the frequency-dependent functional derivative of the KS exchange-correlation potential with respect to the electron density. The quality of the approximations for the exchange-correlation potential and kernel determines the accuracy of the KS based RPA approaches. Initially conventional exchange-correlation densityfunctionals within the local density or generalized gradient approximation were employed in RPA methods [64] and the frequency-dependence of the kernel is neglected, an approximation called adiabatic approximation. Recently methods were introduced $[52,53,55,65]$ that neglect the correlation contribution to the potential and kernel but use the exact exchange potential and the exact frequency-dependent exchange kernel [66-68]. Such methods were named exact exchange (EXX) RPA methods. Hellgren et al. [65] have used this approach to study the correlation ener- 
gies for some atoms using a cubic spline representation for the radial basis sets and found a very good agreement with accurate configuration interaction results. In Ref. [52] Hellgren et al. also investigate a self-consistent exact-exchange RPA method in which the EXX potential is accompanied by a correlation potential derived from an (approximate) functional derivative of the RPA correlation functional. While this led to only small changes for atomic correlation energies compared to the noniterative approach, clear improvements were obtained for the exchange-correlation potentials and thus single-particle spectrum in the KS calculations.

In Ref. [53] we presented the first exact Kohn-Sham exchange RPA method that could be generally applied to molecular systems using an exchange kernel which was derived from a reformulation of the TDDFT response equations omitting the numerically problematic inverses of the noninteracting response functions [69-71]. This new method, termed as EXX-RPA (note that the acronym RPA(EXX) was used in Ref. [53]), has been shown to yield accurate correlation energies and chemical reaction energies if compared to coupled-cluster singles doubles with perturbative triples reference results. In Ref. [55] we have furthermore shown that this EXXRPA correlation functional also correctly describes the bond dissociation of the hydrogen molecule in the asymptotic limit in contrast to the corresponding $\mathrm{HF}$ based adiabatic-connection RPA method. Therefore RPA methods based on the exact KS exchange kernel offer new correlation methods that surpass the accuracy of common density functionals.

While the RPA method including electron exchange effects was originally only defined using Hartree-Fock reference states, in this work it will be shown that the corresponding RPA method on the basis of exact KS exchange (EXX), the EXXRPA method, directly relates to the adiabatic-connection fluctuation dissipation theorem of the KS formalism. The ansatz to determine the KS correlation energy via the adiabatic-connection fluctuation-dissipation theorem, which leads to methods commonly denoted KS based RPA methods, is an exact one, approximations then are made if the exchange-correlation potential and kernel required within this ansatz are chosen. In the EXX-RPA case the approximation of neglecting correlation in potential and kernel is made as only approximation. In HF based RPA methods, on the other hand, the ansatz itself is an approximation, see section 3.1. Moreover, HF based RPA approaches that invoke a coupling constant integration lack a straightforward formal justification for performing this integration because an adiabatic connection between the reference wave function, the HF determinant, and the exact wave function, can not be straightforwardly constructed in a simple way in this case. A further general differences between KS and HF based RPA methods, that shall be elucidated within this work, is the fact that within KS based RPA methods due to their root in the adiabatic-connection fluctuation-dissipation theorem only singulet-singulet excitations need to be considered whereas RPA methods on the basis of HF often require to also consider singulet-triplet exciations.

This work is organized as follows. In section 2 KS RPA methods, i.e., methods that calculate the KS correlation energy via the adiabatic-connection fluctuationdissipation theorem will be introduced. In subsection 2.1 of section 2 we consider the integration of response functions along imaginary frequencies leading to the basic fluctuation-dissipation theorem. In subsection 2.2 the adiabatic connection and the coupling strength integration of the $\mathrm{KS}$ formalism are presented and in subsection 2.3 the coupling strength integration and the fluctuation-dissipation theorem are combined to the adiabatic-connection fluctuation-dissipation theorem. Finally in the last subsection of section 2 the EXX-RPA method is introduced. In section 3 we will then review 'standard' RPA methods that include exchange interactions and that are based on the HF reference determinant. In the first subsection of 
section 3 the general relation between reponse functions and correlation energies is discussed and in this way the formal basis of HF based RPA methods is analysed. In the second subsection of section 3 various variants of HF based RPA methods are introduced and related to perturbation theory. In section 4 relations between HF based RPA methods and the EXX-RPA method are discussed and approaches combining elements from both types of methods are considered. An analysis of results from the various RPA methods for total energies and chemical reaction energies is given in sections 5 and 6 , respectively. Section 7 summarises the results.

\section{RPA correlation energy in density-functional theory}

\subsection{Integration of response functions along imaginary frequencies}

The causal response function $\chi$ that yields the response of the electron density of an electronic system in its ground state with wave function $\Psi_{0}$ on a frequencydependent perturbation is given by

$$
\begin{array}{r}
\chi\left(\mathbf{r}, \mathbf{r}^{\prime}, \nu\right)=\sum_{n \neq 0}\left[\frac{\left\langle\Psi_{0}|\hat{\rho}(\mathbf{r})| \Psi_{n}\right\rangle\left\langle\Psi_{n}\left|\hat{\rho}\left(\mathbf{r}^{\prime}\right)\right| \Psi_{0}\right\rangle}{E_{0}-E_{n}+\nu}\right. \\
\left.+\frac{\left\langle\Psi_{n}|\hat{\rho}(\mathbf{r})| \Psi_{0}\right\rangle\left\langle\Psi_{0}\left|\hat{\rho}\left(\mathbf{r}^{\prime}\right)\right| \Psi_{n}\right\rangle}{E_{0}-E_{n}-\nu}\right] .
\end{array}
$$

The variable $\nu=\omega+i \eta$ shall combine the real-valued frequency $\omega$ of the perturbation and the imaginary convergence factor $i \eta$. In practical applications the limit $\eta \rightarrow 0$ of a vanishing convergence factor is considered. The summation in Eq. (2) runs over all excited states $\Psi_{n}$. The density operator $\hat{\rho}(\mathbf{r})$ is given by

$$
\hat{\rho}(\mathbf{r})=\sum_{i=1}^{N} \delta\left(\mathbf{r}_{i}-\mathbf{r}\right)
$$

with $N$ denoting the number of electrons and $\delta$ designating the delta function. For real-valued Hamiltonian operators the eigenfunctions $\Psi_{n}$ can be chosen real-valued. In this case Eq. (2) for the response function turns into

$$
\chi\left(\mathbf{r}, \mathbf{r}^{\prime}, \nu\right)=-2 \sum_{n \neq 0} \frac{E_{n}-E_{0}}{\left(E_{n}-E_{0}\right)^{2}-\nu^{2}}\left\langle\Psi_{0}|\hat{\rho}(\mathbf{r})| \Psi_{n}\right\rangle\left\langle\Psi_{n}\left|\hat{\rho}\left(\mathbf{r}^{\prime}\right)\right| \Psi_{0}\right\rangle .
$$

Next we consider an integration of the response function with respect to the variable $\nu$ along the imaginary axis by integrating along an integration variable $\omega$ and setting $\nu=i \omega$. With the integral $\int d \omega a / a^{2}+\omega^{2}=\arctan (\omega / a)$ implying

$$
\int_{0}^{\infty} d \omega \frac{\omega}{\omega^{2}+a^{2}}=\frac{\pi}{2} \quad \text { for } \quad a>0
$$


follows

$$
\begin{aligned}
& \frac{-1}{2 \pi} \int_{0}^{\infty} d \omega \chi\left(\mathbf{r}, \mathbf{r}^{\prime}, i \omega\right)= \frac{1}{2} \sum_{n \neq 0}\left\langle\Psi_{0}|\hat{\rho}(\mathbf{r})| \Psi_{n}\right\rangle\left\langle\Psi_{n}\left|\hat{\rho}\left(\mathbf{r}^{\prime}\right)\right| \Psi_{0}\right\rangle \\
&= \frac{1}{2} \sum_{n}\left\langle\Psi_{0}|\hat{\rho}(\mathbf{r})| \Psi_{n}\right\rangle\left\langle\Psi_{n}\left|\hat{\rho}\left(\mathbf{r}^{\prime}\right)\right| \Psi_{0}\right\rangle \\
&-\frac{1}{2}\left\langle\Psi_{0}|\hat{\rho}(\mathbf{r})| \Psi_{0}\right\rangle\left\langle\Psi_{0}\left|\hat{\rho}\left(\mathbf{r}^{\prime}\right)\right| \Psi_{0}\right\rangle \\
&= \frac{1}{2}\left\langle\Psi_{0}\left|\hat{\rho}(\mathbf{r}) \hat{\rho}\left(\mathbf{r}^{\prime}\right)\right| \Psi_{0}\right\rangle-\frac{1}{2} \rho(\mathbf{r}) \rho\left(\mathbf{r}^{\prime}\right) \\
&= \rho_{2}\left(\mathbf{r}, \mathbf{r}^{\prime}\right)-\frac{1}{2} \rho(\mathbf{r}) \rho\left(\mathbf{r}^{\prime}\right) \\
&+\frac{1}{2} \int d \mathbf{r}_{1} d \mathbf{r}_{2} \ldots d \mathbf{r}_{N} d s_{1} d s_{2} \ldots d s_{N} \Psi_{0}\left(\mathbf{r}_{1} \mathbf{r}_{2} \ldots \mathbf{r}_{N}\right) \\
& \times\left[\sum_{i=1}^{N} \delta\left(\mathbf{r}_{i}-\mathbf{r}\right) \delta\left(\mathbf{r}_{i}-\mathbf{r}^{\prime}\right)\right] \Psi_{0}\left(\mathbf{r}_{1} \mathbf{r}_{2} \ldots \mathbf{r}_{N}\right) .(6)
\end{aligned}
$$

In Eq. (6), by $d s_{1} d s_{2} \ldots d s_{N}$ the integration over spin variables shall be denoted, $\rho_{2}\left(\mathbf{r}, \mathbf{r}^{\prime}\right)$ designates the pair density, the diagonal of the second order spinless density matrix defined according to

$$
\begin{aligned}
\rho_{2}\left(\mathbf{r}, \mathbf{r}^{\prime}\right)=\frac{1}{2} \int d \mathbf{r}_{1} d \mathbf{r}_{2} \ldots d \mathbf{r}_{N} d s_{1} d s_{2} \ldots d s_{N} \Psi_{0}\left(\mathbf{r}_{1} \mathbf{r}_{2} \ldots \mathbf{r}_{N}\right) \\
\times\left[\sum_{\substack{i=1 \\
N}}^{N} \sum_{\substack{j=1 \\
j \neq i}}^{N} \delta\left(\mathbf{r}_{i}-\mathbf{r}\right) \delta\left(\mathbf{r}_{j}-\mathbf{r}^{\prime}\right)\right] \Psi_{0}\left(\mathbf{r}_{1} \mathbf{r}_{2} \ldots \mathbf{r}_{N}\right) .
\end{aligned}
$$

In the step from the second to the third equality of Eq. (6) it is exploited that $\sum_{n}\left|\Psi_{n}\right\rangle\left\langle\Psi_{n}\right|$ represents the identity operator.

Next we multiply Eq. (6) by a function $g\left(\mathbf{r}, \mathbf{r}^{\prime}\right)$ which, at this point, shall be arbitrary and later will be set equal to $1 /\left|\mathbf{r}-\mathbf{r}^{\prime}\right|$. Furthermore we integrate over 
the variables $\mathbf{r}$ and $\mathbf{r}^{\prime}$

$$
\begin{aligned}
\frac{-1}{2 \pi} \int_{0}^{\infty} d \omega \int d \mathbf{r} d \mathbf{r}^{\prime} g\left(\mathbf{r}, \mathbf{r}^{\prime}\right) \chi\left(\mathbf{r}, \mathbf{r}^{\prime}, i \omega\right) & \\
= & \int d \mathbf{r} d \mathbf{r}^{\prime} g\left(\mathbf{r}, \mathbf{r}^{\prime}\right)\left[\rho_{2}\left(\mathbf{r}, \mathbf{r}^{\prime}\right)-\frac{1}{2} \rho(\mathbf{r}) \rho\left(\mathbf{r}^{\prime}\right)\right] \\
& +\int d \mathbf{r} d \mathbf{r}^{\prime} \frac{1}{2} \int d \mathbf{r}_{1} d \mathbf{r}_{2} \ldots d \mathbf{r}_{N} d s_{1} d s_{2} \ldots d s_{N} \Psi_{0}\left(\mathbf{r}_{1} \mathbf{r}_{2} \ldots \mathbf{r}_{N}\right) \\
& \times g\left(\mathbf{r}, \mathbf{r}^{\prime}\right)\left[\sum_{i=1}^{N} \delta\left(\mathbf{r}_{i}-\mathbf{r}\right) \delta\left(\mathbf{r}_{i}-\mathbf{r}^{\prime}\right)\right] \Psi_{0}\left(\mathbf{r}_{1} \mathbf{r}_{2} \ldots \mathbf{r}_{N}\right) \\
= & \int d \mathbf{r} d \mathbf{r}^{\prime} g\left(\mathbf{r}, \mathbf{r}^{\prime}\right)\left[\rho_{2}\left(\mathbf{r}, \mathbf{r}^{\prime}\right)-\frac{1}{2} \rho(\mathbf{r}) \rho\left(\mathbf{r}^{\prime}\right)\right] \\
& +\int d \mathbf{r} d \mathbf{r}^{\prime} \frac{1}{2} \int d \mathbf{r}_{1} d \mathbf{r}_{2} \ldots d \mathbf{r}_{N} d s_{1} d s_{2} \ldots d s_{N} \Psi_{0}\left(\mathbf{r}_{1} \mathbf{r}_{2} \ldots \mathbf{r}_{N}\right) \\
& \times g\left(\mathbf{r}, \mathbf{r}^{\prime}\right)\left[\sum_{i=1}^{N} \delta\left(\mathbf{r}_{i}-\mathbf{r}\right) \delta\left(\mathbf{r}^{\prime}-\mathbf{r}\right)\right] \Psi_{0}\left(\mathbf{r}_{1} \mathbf{r}_{2} \ldots \mathbf{r}_{N}\right) \\
= & \int d \mathbf{r} d \mathbf{r}^{\prime} g\left(\mathbf{r}, \mathbf{r}^{\prime}\right)\left[\rho_{2}\left(\mathbf{r}, \mathbf{r}^{\prime}\right)-\frac{1}{2} \rho(\mathbf{r}) \rho\left(\mathbf{r}^{\prime}\right)\right] \\
& +\frac{1}{2} \int d \mathbf{r} d \mathbf{r}^{\prime} g\left(\mathbf{r}, \mathbf{r}^{\prime}\right) \rho(\mathbf{r}) \delta\left(\mathbf{r}-\mathbf{r}^{\prime}\right) .
\end{aligned}
$$

In the step form the first to the second equality in Eq. (8) we used the relation

$$
\begin{aligned}
\int d \mathbf{r} d \mathbf{r}^{\prime} d \mathbf{r}_{i} g\left(\mathbf{r}, \mathbf{r}^{\prime}\right) f\left(\mathbf{r}_{i}\right) \delta\left(\mathbf{r}_{i}-\mathbf{r}\right) \delta\left(\mathbf{r}_{i}-\mathbf{r}^{\prime}\right) & =\int d \mathbf{r} d \mathbf{r}_{i} g\left(\mathbf{r}, \mathbf{r}_{i}\right) f\left(\mathbf{r}_{i}\right) \delta\left(\mathbf{r}_{i}-\mathbf{r}\right) \\
& =\int d \mathbf{r}_{i} g\left(\mathbf{r}_{i}, \mathbf{r}_{i}\right) f\left(\mathbf{r}_{i}\right) \\
& =\int d \mathbf{r} g(\mathbf{r}, \mathbf{r}) f(\mathbf{r}) \\
& =\int d \mathbf{r} d \mathbf{r}_{i} g(\mathbf{r}, \mathbf{r}) f\left(\mathbf{r}_{i}\right) \delta\left(\mathbf{r}_{i}-\mathbf{r}\right) \\
& =\int d \mathbf{r} d \mathbf{r}^{\prime} d \mathbf{r}_{i} g\left(\mathbf{r}, \mathbf{r}^{\prime}\right) f\left(\mathbf{r}_{i}\right) \delta\left(\mathbf{r}_{i}-\mathbf{r}\right) \delta\left(\mathbf{r}^{\prime}-\mathbf{r}\right)
\end{aligned}
$$

which holds true for arbitrary functions $f\left(\mathbf{r}_{i}\right)$ and thus also for $f\left(\mathbf{r}_{i}\right)=$ $\Psi_{0}\left(\mathbf{r}_{1} \mathbf{r}_{2} \ldots \mathbf{r}_{i} \ldots \mathbf{r}_{N}\right) \Psi_{0}\left(\mathbf{r}_{1} \mathbf{r}_{2} \ldots \mathbf{r}_{i} \ldots \mathbf{r}_{N}\right)$.

For $g(\mathbf{r}, \mathbf{r})=1 /\left|\mathbf{r}-\mathbf{r}^{\prime}\right|$ the first integral on the right hand side of equation (8) yields

$$
\int d \mathbf{r} d \mathbf{r}^{\prime} \frac{1}{\left|\mathbf{r}-\mathbf{r}^{\prime}\right|}\left[\rho_{2}\left(\mathbf{r}, \mathbf{r}^{\prime}\right)-\frac{1}{2} \rho(\mathbf{r}) \rho\left(\mathbf{r}^{\prime}\right)\right]=V_{e e}-U
$$




\section{Molecular Physics}

i.e., the sum of the electron-electron interaction energy

$$
V_{e e}=\int d \mathbf{r} d \mathbf{r}^{\prime} \frac{\rho_{2}\left(\mathbf{r}, \mathbf{r}^{\prime}\right)}{\left|\mathbf{r}-\mathbf{r}^{\prime}\right|}=\left\langle\Psi_{0}\left|\hat{V}_{e e}\right| \Psi_{0}\right\rangle
$$

with the operator of the electron-electron interaction given by

$$
\hat{V}_{e e}=\sum_{i=1}^{N} \sum_{\substack{j=1 \\ j \neq i}}^{N} \frac{1}{\left|\mathbf{r}_{i}-\mathbf{r}_{j}\right|}
$$

minus the Coulomb energy

$$
U=\frac{1}{2} \int d \mathbf{r} d \mathbf{r}^{\prime} \frac{\rho(\mathbf{r}) \rho\left(\mathbf{r}^{\prime}\right)}{\left|\mathbf{r}-\mathbf{r}^{\prime}\right|} .
$$

The second integral on the right hand side of equation (8) diverges for $g(\mathbf{r}, \mathbf{r})=$ $1 /\left|\mathbf{r}-\mathbf{r}^{\prime}\right|$. Later on we will consider differences of expressions of the form given on the right hand side of Eq. (8). In these differences the divergent contributions will cancel each other and therefore will not lead to problems.

\subsection{Coupling strength integration}

An adiabatic connection $[28,72,73]$ between the Kohn-Sham model system, a system of hypothetical noninteracting electrons, and the corresponding real electronic system is defined with the help of the Schrödinger equation

$$
\left[\hat{T}+\alpha \hat{V}_{e e}+\hat{v}(\alpha)\right] \Psi_{0}(\alpha)=E_{0}(\alpha) \Psi_{0}(\alpha)
$$

that contains a coupling constant $\alpha$ with $0 \leq \alpha \leq 1$ scaling the electron-electron interaction operator $\hat{V}_{e e}$ defined in Eq. (12) and a coupling-constant-dependent potential

$$
\hat{v}(\alpha)=\sum_{i}^{N} v\left(\alpha, \mathbf{r}_{i}\right) .
$$

The operator of the kinetic energy in Eq. (14) is given by

$$
\hat{T}=-\sum_{i}^{N} \frac{1}{2} \nabla_{i}^{2}
$$

The potential $v\left(\alpha, \mathbf{r}_{i}\right)$ is defined up to an additive constant by the requirement that the ground state wave functions $\Psi_{0}(\alpha)$ for all values of the coupling constant $\alpha$ yield the same electron density $\rho(\mathbf{r})$, i.e.,

$$
\left\langle\Psi_{0}(\alpha)|\hat{\rho}(\mathbf{r})| \Psi_{0}(\alpha)\right\rangle=\rho(\mathbf{r}) .
$$

The Hohenberg-Kohn theorem guarantees that up to an additive constant the potential $v\left(\alpha, \mathbf{r}_{i}\right)$ is uniquely defined by this density condition. For vanishing coupling 
constants the potential $v\left(\alpha, \mathbf{r}_{i}\right)$ equals the effective $\mathrm{KS}$ potential $v_{s}(\mathbf{r})$, i.e.,

$$
v(\alpha=0, \mathbf{r})=v_{s}(\mathbf{r})
$$

and for a coupling constant $\alpha=1$ the potential $v\left(\alpha, \mathbf{r}_{i}\right)$ equals the external potential

$$
v(\alpha=1, \mathbf{r})=v_{e x t}(\mathbf{r})
$$

of the considered real electron system, usually the electrostatic potential of the nuclei.

The eigenfunctions $\Psi_{n}(\alpha)$ of the adiabatic connection Schrödinger equation (14), for a vanishing coupling constant $\alpha=0$, are the ground and excited KS wave functions $\Phi_{n}$,

$$
\Psi_{n}(\alpha=0)=\Phi_{n}
$$

and, for coupling constant $\alpha=1$, equal the eigenstates $\Psi_{n}$ of the real electron system

$$
\Psi_{n}(\alpha=1)=\Psi_{n}
$$

Note that the Hohenberg-Kohn theorem guarantess only the uniqueness of the coupling-constant-dependent potentials $v(\alpha, \mathbf{r})$ but not their existence. For the fully interacting case, i.e., $\alpha=1$, the potential $v(\alpha=1, \mathbf{r})=v_{\text {ext }}(\mathbf{r})$ is given by the considered real electronic system. For other values of the coupling constant $\alpha$ the existence of $v(\alpha, \mathbf{r})$ has to be assumed, an assumption that is called v-representability assumption and that underlies the KS formalism and thus most applications of DFT. The KS formalism only requires that with a given external potential $v(\alpha=1, \mathbf{r})=v_{\text {ext }}(\mathbf{r})$ also the KS potential $v(\alpha=0, \mathbf{r})=v_{s}(\mathbf{r})$ exists, for an adiabatic connection as it is defined here potentials $v(\alpha, \mathbf{r})$ for all values $0 \leq \alpha \leq 1$ need to exist.

The ground state wave function $\Phi_{0}$ of the KS system defines the noninteracting kinetic energy

$$
T_{s}=\left\langle\Phi_{0}|\hat{T}| \Phi_{0}\right\rangle
$$

by its expectation value with the kinetic energy operator and the KS exchange energy

$$
E_{x}=\left\langle\Phi_{0}\left|\hat{V}_{e e}\right| \Phi_{0}\right\rangle-U
$$

by its expectation value with the electron-electron interation operator minus the Coulomb energy $U$ of Eq. (13). Both the noninteracting kinetic energy as well as the exchange energy only depend on the KS wave function $\Phi_{0}$ and thus are independent of the coupling constant $\alpha$. That is $T_{s}, E_{x}$, and $U$ are given by the starting point of the adiabatic connection at $\alpha=0$. The definition of the KS exchange energy, i.e., the exchange energy in DFT, thus differs from the definition of the exchange energy as it is common in wave function based methods. In the latter case the exchange energy is defined as the electron-electron interaction energy of the HF determinant minus the Coulomb energy $U$. For nondegenerate ground states the KS wave function $\Phi_{0}$ like the HF wave function is a single Slater determinant. The exchange energy then in both cases is given by the well-kown expression for the 
exchange energy of a Slater determinant in terms of the orbitals building the Slater determinant. However, the orbitals entering the expression in the one case are KS orbitals and in the other are HF orbitals and therefore the values of the KS and HF exchange energies are different. In practice the differences are small because KS and HF determinants turn out to be surprisingly similar [70, 74-76].

A coupling-constant-dependent correlation energy $E_{c}(\alpha)$ shall be defined according to

$$
E_{c}(\alpha)=\left\langle\Psi_{0}(\alpha)\left|\hat{T}+\alpha \hat{V}_{e e}\right| \Psi_{0}(\alpha)\right\rangle-\left\langle\Phi_{0}\left|\hat{T}+\alpha \hat{V}_{e e}\right| \Phi_{0}\right\rangle
$$

The correlation energy $E_{c}=E_{c}(\alpha=1)$ for $\alpha=1$ is the KS or DFT correlation energy which differs in its definition and its value from the correlation energy as it is commonly defined in wave function based methods. Because, by construction, the wave functions $\Psi_{0}(\alpha)$ and $\Phi_{0}$ that enter the definition of the correlation energy $E_{c}(\alpha)$ yield the same electron density we can turn Eq. (24) into

$$
E_{c}(\alpha)=\left\langle\Psi_{0}(\alpha)\left|\hat{T}+\alpha \hat{V}_{e e}+\hat{v}(\alpha)\right| \Psi_{0}(\alpha)\right\rangle-\left\langle\Phi_{0}\left|\hat{T}+\alpha \hat{V}_{e e}+\hat{v}(\alpha)\right| \Phi_{0}\right\rangle .
$$

For $\alpha=1 \mathrm{Eq}$. (25) yields the KS or DFT correlation energy as the difference of the expectation values of the electronic Hamiltonian operator with the full ground state wave function $\Psi_{0}=\Psi_{0}(\alpha=1)$, i.e. the full ground state energy, minus the expectation values of the electronic Hamiltonian operator with the KS wave function $\Phi_{0}=\Psi_{0}(\alpha=0)$. The HF based correlation energy, on the other hand, is the difference of the expectation values of the electronic Hamiltonian operator with the full ground state wave function and the HF determinant.

The correlation energy $E_{c}(\alpha)$ of Eq. (24) is the sum

$$
E_{c}(\alpha)=T_{c}(\alpha)+\alpha V_{c}(\alpha)
$$

of a kinetic contribution,

$$
T_{c}(\alpha)=\left\langle\Psi_{0}(\alpha)|\hat{T}| \Psi_{0}(\alpha)\right\rangle-\left\langle\Phi_{0}|\hat{T}| \Phi_{0}\right\rangle
$$

and an electron-electron interaction contribution

$$
V_{c}(\alpha)=\left\langle\Psi_{0}(\alpha)\left|\hat{V}_{e e}\right| \Psi_{0}(\alpha)\right\rangle-\left\langle\Phi_{0}\left|\hat{V}_{e e}\right| \Phi_{0}\right\rangle
$$

multiplied by the coupling constant $\alpha$.

The derivative of the correlation energy $E_{c}(\alpha)$ with respect to the coupling constant is given by $V_{c}(\alpha)$ because taking the derivative of Eq. (25) yields

$$
\begin{aligned}
\frac{d E_{c}(\alpha)}{d \alpha}= & \left\langle\Psi_{0}(\alpha)\left|\hat{V}_{e e}+\frac{d \hat{v}(\alpha)}{d \alpha}\right| \Psi_{0}(\alpha)\right\rangle-\left\langle\Phi_{0}\left|\hat{V}_{e e}+\frac{d \hat{v}(\alpha)}{d \alpha}\right| \Phi_{0}\right\rangle \\
= & \left\langle\Psi_{0}(\alpha)\left|\hat{V}_{e e}\right| \Psi_{0}(\alpha)\right\rangle+\int d \mathbf{r} \frac{d v(\alpha, \mathbf{r})}{d \alpha} \rho(\mathbf{r}) \\
& \quad-\left\langle\Phi_{0}\left|\hat{V}_{e e}\right| \Phi_{0}\right\rangle-\int d \mathbf{r} \frac{d v(\alpha, \mathbf{r})}{d \alpha} \rho(\mathbf{r}) \\
= & \left\langle\Psi_{0}(\alpha)\left|\hat{V}_{e e}\right| \Psi_{0}(\alpha)\right\rangle-\left\langle\Phi_{0}\left|\hat{V}_{e e}\right| \Phi_{0}\right\rangle \\
= & V_{c}(\alpha)
\end{aligned}
$$


For the first line of Eq. (29) the Hellmann-Feynman theorem was invoked, for the second line it was exploited that $\Psi_{0}(\alpha)$ as well as $\Phi_{0}$ yield the electron density $\rho$.

From Eq. (29) follows immediately the coupling strengths integration for the DFT correlation energy $E_{c}=E_{c}(\alpha=1)$,

$$
\begin{aligned}
E_{c} & =E_{c}(\alpha=1) \\
& =E_{c}(\alpha=1)-E_{c}(\alpha=0) \\
& =\int_{0}^{1} d \alpha \frac{d E_{c}(\alpha)}{d \alpha} \\
& =\int_{0}^{1} d \alpha V_{c}(\alpha),
\end{aligned}
$$

if we exploit that definition (24) of the correlation energy $E_{c}(\alpha)$ implies $E_{c}(\alpha=0)=0$.

\subsection{Adiabatic-connection fluctuation-dissipation theorem}

By combining the coupling strength integration, Eq. (30), with the integration of causal response functions along complex frequencies, Eq. (8), we obtain the adiabatic connection fluctuation dissipation theorem for the DFT correlation energy $E_{c}$ $[28,29,35,64,77]$. In Eq. (8) the integration of the response function $\chi\left(\mathbf{r}, \mathbf{r}^{\prime}, i \omega\right)$ of the real electron system is considered. A generalization to a coupling-strengthdependent response function $\chi\left(\alpha, \mathbf{r}, \mathbf{r}^{\prime}, i \omega\right)$ is straightforward by replacing the wave functions $\Psi_{n}$ in Eqs. (2) - (11) by the wave functions $\Psi_{n}(\alpha)$ and by introducing the coupling-strength dependent pair density $\rho_{2}\left(\alpha, \mathbf{r}, \mathbf{r}^{\prime}\right)$, the diagonal of a couplingstrength-dependent second order spinless density matrix obtained by a generalization of definition (7). The coupling strength integration for $E_{c}$ then can be expressed as

$$
\begin{aligned}
E_{c}= & \int_{0}^{1} d \alpha V_{c}(\alpha) \\
= & \int_{0}^{1} d \alpha\left\langle\Psi_{0}(\alpha)\left|\hat{V}_{e e}\right| \Psi_{0}(\alpha)\right\rangle-\left\langle\Phi_{0}\left|\hat{V}_{e e}\right| \Phi_{0}\right\rangle \\
= & \int_{0}^{1} d \alpha \int d \mathbf{r} d \mathbf{r}^{\prime} \frac{\rho_{2}\left(\alpha, \mathbf{r}, \mathbf{r}^{\prime}\right)}{\left|\mathbf{r}-\mathbf{r}^{\prime}\right|}-\frac{\rho_{2}\left(\alpha=0, \mathbf{r}, \mathbf{r}^{\prime}\right)}{\left|\mathbf{r}-\mathbf{r}^{\prime}\right|} \\
= & \int_{0}^{1} d \alpha \int d \mathbf{r} d \mathbf{r}^{\prime}\left[\frac{\rho_{2}\left(\alpha, \mathbf{r}, \mathbf{r}^{\prime}\right)}{\left|\mathbf{r}-\mathbf{r}^{\prime}\right|}-\frac{1}{2} \frac{\rho(\mathbf{r}) \rho\left(\mathbf{r}^{\prime}\right)}{\left|\mathbf{r}-\mathbf{r}^{\prime}\right|}\right. \\
& \left.\quad+\frac{\rho_{2}\left(\alpha=0, \mathbf{r}, \mathbf{r}^{\prime}\right)}{\left|\mathbf{r}-\mathbf{r}^{\prime}\right|}-\frac{1}{2} \frac{\rho(\mathbf{r}) \rho\left(\mathbf{r}^{\prime}\right)}{\left|\mathbf{r}-\mathbf{r}^{\prime}\right|}\right] .
\end{aligned}
$$

Only one density $\rho$ that is independent of $\alpha$ occurs in Eq. (31) because the wave functions $\Psi_{0}(\alpha)$ independently of $\alpha$ yield the same electron density $\rho$ If we now insert twice Eq. (8) with $g\left(\mathbf{r}, \mathbf{r}^{\prime}\right)=1 /\left|\mathbf{r}-\mathbf{r}^{\prime}\right|$ and use that the last integral in Eq. (8) is cancelled because it occurs twice with different signs then we obtain the adiabatic-connection fluctuation-dissipation theorem for the DFT correlation 
energy $E_{c}$

$$
E_{c}=\frac{-1}{2 \pi} \int_{0}^{1} d \alpha \int d \mathbf{r} d \mathbf{r}^{\prime} \frac{1}{\left|\mathbf{r}-\mathbf{r}^{\prime}\right|} \int_{0}^{\infty} d \omega\left[\chi_{\alpha}\left(\mathbf{r}, \mathbf{r}^{\prime}, i \omega\right)-\chi_{0}\left(\mathbf{r}, \mathbf{r}^{\prime}, i \omega\right)\right]
$$

\subsection{RPA correlation energy within Kohn-Sham framework}

In order to calculate the Kohn-Sham correlation energy with the adiabaticconnection fluctuation-dissipation theorem the response functions $\chi_{\alpha}$ and $\chi_{0}$ are required. The KS response function $\chi_{0}$ is known in terms of the occupied and unoccupied KS orbitals $\varphi_{i}$ and $\varphi_{a}$, respectively, and their eigenvalues $\varepsilon_{i}$ and $\varepsilon_{a}$,

$$
\chi_{0}\left(\mathbf{r}, \mathbf{r}^{\prime}, i \omega\right)=\sum_{i}^{\text {occ. unocc. }} \sum_{a} \frac{-4 \varepsilon_{i a}}{\varepsilon_{i a}^{2}+\omega^{2}} \varphi_{i}(\mathbf{r}) \varphi_{a}(\mathbf{r}) \varphi_{a}\left(\mathbf{r}^{\prime}\right) \varphi_{i}\left(\mathbf{r}^{\prime}\right) .
$$

In Eq.(33) $\varepsilon_{i a}=\varepsilon_{a}-\varepsilon_{i}$ and thus by definition is a positive quantity. Throughout this Section we consider non-sin-polarized systems. By $\varphi_{i}$ and $\varphi_{a}$ therefore spatial orbitals are denoted while the spin degree of freedom is taken into account by appropriate prefactors. Throughout this work indices $i$ and $j$ denote occupied, $a$ and $b$ unoccupied, and $p, q, r$, and $s$ arbitray orbitals. Summations over the indices run over the occupied, the unoccupied, or all orbitals, respectively.

The integration over the frequency and the spatial variables is straightforward and leads to

$$
\frac{-1}{2 \pi} \int d \mathbf{r} d \mathbf{r}^{\prime} \frac{1}{\left|\mathbf{r}-\mathbf{r}^{\prime}\right|} \int_{0}^{\infty} d \omega \chi_{0}\left(\mathbf{r}, \mathbf{r}^{\prime}, i \omega\right)=\sum_{i a}\langle i a \mid a i\rangle=\operatorname{Tr}[\mathbf{C}]
$$

with $\langle i a \mid a i\rangle$ denoting a two-electron integral in physicist's notation and $\mathbf{C}$ designating a matrix defined by the matrix elements $C_{i a, j b}=\langle i b \mid a j\rangle$. The dimension of the matrix $\mathbf{C}$ equals the number of occupied times unoccupied KS orbitals, its columns and rows are labeled by the superindices $j b$ and $i a$.

The coupling-strength-dependent response function $\chi_{\alpha}$ is obtained by timedependent DFT (TDDFT) in the linear response regime. The basic equation of time-dependent or more precisely frequency-dependent KS response theory is [7883].

$$
\left[\varepsilon^{2}+\varepsilon^{1 / 2} \mathbf{K}_{\mathrm{uxc}}(\alpha, \omega) \varepsilon^{1 / 2}\right] \mathbf{z}_{n}(\alpha, \omega)=\Omega_{n}^{2}(\alpha, \omega) \mathbf{z}_{n}(\alpha, \omega) .
$$

In Eq. (35) $\varepsilon$ designates a diagonal matrix with elements $\varepsilon_{i a, j b}=\delta_{i a, j b} \varepsilon_{i a}=$ $\delta_{i a, j b}\left(\varepsilon_{a}-\varepsilon_{i}\right)$. The kernel matrix $\mathbf{K}_{\mathrm{uxc}}(\alpha, \omega)$ is defined by its matrix elements $\int d \mathbf{r} d \mathbf{r}^{\prime} \varphi_{i}(\mathbf{r}) \varphi_{a}(\mathbf{r}) f_{u x c}\left(\alpha, \omega, \mathbf{r}, \mathbf{r}^{\prime}\right) \varphi_{a}\left(\mathbf{r}^{\prime}\right) \varphi_{i}\left(\mathbf{r}^{\prime}\right)$. The kernel $f_{u x c}$ is the frequency- and coupling-strength-dependent functional derivative of the sum of the Hartree and KS exchange-correlation potential.

Eq. (35) is an equation that is nonlinear in the frequency $\omega$. For a given frequency $\omega$ the number of eigenvalues $\Omega_{n}(\alpha, \omega)^{2}$ equals the product of occupied times unoccupied orbitals. However, only if a square root $\Omega_{n}(\alpha, \omega)$ equals the frequency $\omega$ then this $\Omega_{n}(\alpha, \omega)$ equals an exitation energy. In most TDDFT methods the frequency-dependence of the kernel is neglected, i.e., the adiabatic approximation is employed. In this case the frequency-dependenc of the kernel and the eigenvectors $\mathbf{z}_{n}$ vanishes, Eq. (35) becomes linear, and the square roots $\Omega_{n}$ of the eigenvalues equal the excitation energies. In this work, however, we construct the response 
function $\chi_{\alpha}$ within an exact-exchange TDDFT (TDEXX) framework, that is we neglect the correlation contribution to the kernel $f_{u x c}$ but we treat the exchange contribution exactly including its frequency dependence. The exact exchange kernel is given by a quite complicated integral expression [66-68] which is not well suited for numerical implementations. It is, however, possible to derive an equation for the frequency-dependent response of the effective KS potential and not, as usually in TDDFT, of the electron density [69]. The corresponding eigenvalue equation for the excitation energies is better suited for an exact treatment of exchange. At first, the eigenvalue equation is again nonlinear in the frequency $\omega$ but it can be rearranged into the linear generalized eigenvalue equation [71], the TDEXX equation

$$
\begin{aligned}
& {\left[\varepsilon^{2}+\alpha \varepsilon^{1 / 2}[\mathbf{A}+\mathbf{B}+\boldsymbol{\Delta}] \varepsilon^{1 / 2}\right] \mathbf{z}_{n}(\alpha)=} \\
& \Omega_{n}^{2}(\alpha)\left[\mathbf{1}-\alpha \boldsymbol{\varepsilon}^{-1 / 2}[\mathbf{A}-\mathbf{B}+\boldsymbol{\Delta}] \boldsymbol{\varepsilon}^{-1 / 2}\right] \mathbf{z}_{n}(\alpha) .
\end{aligned}
$$

The matrices $\mathbf{A}, \mathbf{B}, \boldsymbol{\Delta}$ with a dimension equal to the number of occupied times unoccupied KS orbitals contain the matrix elements $A_{i a, j b}=2\langle i j \mid a b\rangle-\langle i a \mid j b\rangle$, $B_{i a, j b}=2\langle i j \mid a b\rangle-\langle i j \mid b a\rangle, \Delta_{i a, j b}=\delta_{i j}\left\langle\varphi_{a}\left|\hat{v}_{\mathrm{x}}^{\mathrm{NL}}-\hat{v}_{\mathrm{x}}\right| \varphi_{b}\right\rangle-\delta_{a b}\left\langle\varphi_{i}\left|\hat{v}_{\mathrm{x}}^{\mathrm{NL}}-\hat{v}_{\mathrm{x}}\right| \varphi_{j}\right\rangle$. The indices $i a$ and $j b$, again, are superindices labeling the rows and columns of the matrices. The operator $\hat{v}_{\mathrm{x}}^{\mathrm{NL}}$ is a nonlocal exchange operator of the form of the HF exchange operator but is constructed from KS orbitals while $\hat{v}_{\mathrm{x}}$ is the operator corresponding to the local multiplicative KS exchange potential. Because the exact exchange kernel as well as the Hartree kernel are linear in the coupling strength $\alpha$, the coupling strength occurs as linear prefactor in the equation. The price for having arranged Eq. (36) in a form that does no longer contain terms depending on $\omega$ is that Eq. (36) in contrast to Eq. (35) is a generalized eigenvalue equation.

With the eigenvectors $\mathbf{z}_{n}(\alpha)$ and the square roots $\Omega_{n}(\alpha)$ of Eq. (36) the response function $\chi_{\alpha}$ can be expressed as [71]

$$
\begin{gathered}
\chi_{\alpha}\left(i \omega, \mathbf{r}, \mathbf{r}^{\prime}\right)=\sum_{n} \frac{1}{\Omega_{n}(\alpha)} \sum_{i a} \sum_{j b} \varphi_{i}(\mathbf{r}) \varphi_{a}(\mathbf{r})\left[\varepsilon^{1 / 2} \mathbf{z}_{n}(\alpha) \frac{-4 \Omega_{n}(\alpha)}{\Omega_{n}(\alpha)^{2}+\omega^{2}} \mathbf{z}_{n}(\alpha)^{T} \varepsilon^{1 / 2}\right]_{i a, j b} \\
\times \varphi_{b}\left(\mathbf{r}^{\prime}\right) \varphi_{j}\left(\mathbf{r}^{\prime}\right)
\end{gathered}
$$

Integration over the frequency and the spatial variables yields

$$
\frac{-1}{2 \pi} \int d \mathbf{r} d \mathbf{r}^{\prime} \frac{1}{\left|\mathbf{r}-\mathbf{r}^{\prime}\right|} \int_{0}^{\infty} d \omega \chi_{\alpha}\left(\mathbf{r}, \mathbf{r}^{\prime}, i \omega\right)=\sum_{n} \mathbf{z}_{n}^{T}(\alpha) \varepsilon^{1 / 2} \mathbf{C} \varepsilon^{1 / 2} \mathbf{z}_{n}(\alpha) / \Omega_{n}(\alpha)
$$

Finally subtracting Eqs. (34) and (38) and integration over the coupling constant $\alpha$ yields the EXX-RPA correlation energy

$$
E_{c}=\int_{0}^{1} d \alpha V_{c}(\alpha)
$$

with

$$
V_{c}(\alpha)=\left[\sum_{n} \mathbf{z}_{n}^{T}(\alpha) \varepsilon^{1 / 2} \mathbf{C} \varepsilon^{1 / 2} \mathbf{z}_{n}(\alpha) / \Omega_{n}(\alpha)\right]-\operatorname{Tr}[\mathbf{C}]
$$




\section{RPA correlation energy based on Hartree-Fock}

\subsection{Response functions and correlation energies in RPA based on Hartree-Fock}

Following Oddershede [84] the total electronic ground-state energy can be written as

$$
\begin{aligned}
E_{0}= & \sum_{p q}\left[h_{p q}+\frac{1}{4} \sum_{r}\langle p r \| q r\rangle+\frac{1}{4} \sum_{r s}\langle p r \| q s\rangle\left\langle\Psi_{0}\left|a_{r}^{\dagger} a_{s}\right| \Psi_{0}\right\rangle\right]\left\langle\Psi_{0}\left|a_{p}^{\dagger} a_{q}\right| \Psi_{0}\right\rangle \\
& -\frac{1}{4 \pi} \sum_{p q r s}\langle p r \| q s\rangle \int_{0}^{\infty} d \omega \chi_{p q, r s}(i \omega)
\end{aligned}
$$

where the summations over $p, q, r$ and $s$ run over all, i.e., occupied and unoccupied spin orbitals. This means in this Section, in contrast to the previous one, we consider orbitals including their spin degree of freedom and summations run over spin orbitals, i.e., indices $p, q$ etc. shall include possible spin indices. By $\langle p q \| r s\rangle$ an antisymmetrised two-electron integral in physicist's notation is denoted, $\hat{a}_{s}$ and $\hat{a}_{q}$ designate annihilation, and $\hat{a}_{r}^{\dagger}$ and $\hat{a}_{p}^{\dagger}$ creation operators. The sum $\sum_{r s}\left\langle\Psi_{0}\left|a_{r}^{\dagger} a_{s}\right| \Psi_{0}\right\rangle$ determines the first order density matrix $\rho\left(\mathbf{r}, \mathbf{r}^{\prime}\right)$ according to $\rho\left(\mathbf{r}, \mathbf{r}^{\prime}\right)=\sum_{r s} \phi_{r}^{\dagger}(\mathbf{r}) \phi_{s}\left(\mathbf{r}^{\prime}\right)\left\langle\Psi_{0}\left|\hat{a}_{r}^{\dagger} \hat{a}_{s}\right| \Psi_{0}\right\rangle$ (likewise the sum $\left.\sum_{p q}\left\langle\Psi_{0}\left|a_{p}^{\dagger} a_{q}\right| \Psi_{0}\right\rangle\right)$. The response matrix elements $\chi_{p q, r s}(\nu)$ define the response function $\sum_{p q r s} \phi_{p}^{\dagger}\left(\mathbf{r}_{2}\right) \phi_{q}\left(\mathbf{r}_{2}^{\prime}\right) \chi_{p q, r s}(\nu) \phi_{r}^{\dagger}\left(\mathbf{r}_{1}\right) \phi_{s}\left(\mathbf{r}_{1}^{\prime}\right)$ that yields the response of the first order density matrix on a perturbation given by a general one-electron operator. That is in constrast to the response function (2) of the previous Section the perturbation can be a nonlocal operator and the response of the first order density matrix not just of the electron density is considered. By $\phi_{p} \phi_{q}, \phi_{r}$, and $\phi_{s}$ spin orbitals are denoted.

The response matrix elements $\chi_{p q, r s}(i \omega)$ are given by

$$
\chi_{p q, r s}(i \omega)=\sum_{n \neq 0}\left[\frac{\left\langle\Psi_{0}\left|\hat{a}_{p}^{\dagger} \hat{a}_{q}\right| \Psi_{n}\right\rangle\left\langle\Psi_{n}\left|\hat{a}_{r}^{\dagger} \hat{a}_{s}\right| \Psi_{0}\right\rangle}{E_{0}-E_{n}+i \omega}+\frac{\left\langle\Psi_{n}\left|\hat{a}_{p}^{\dagger} \hat{a}_{q}\right| \Psi_{0}\right\rangle\left\langle\Psi_{0}\left|\hat{a}_{r}^{\dagger} \hat{a}_{s}\right| \Psi_{n}\right\rangle}{E_{0}-E_{n}-i \omega}\right]
$$

In order to extract the correlation energy from Eq. (41) one may now add and subtract the contribution $-\frac{1}{4 \pi} \sum_{p q r s}\langle p r \| s q\rangle \int_{0}^{\infty} d \omega \chi_{p q, r s}^{0}(i \omega)$ from Eq. (41) where $\chi_{p q, r s}^{0}(i \omega)$ refers to a general single-particle (e.g. Kohn-Sham or Hartree-Fock) response function that is obtained from Eq. (42) by replacing the exact eigenfunctions $\Psi_{0}$ and $\Psi_{n}$ by determinantal wave functions $\Phi_{0}$ and $\Phi_{n}$. In this case the expectation 
values and the summation over $n$ are readily carried out in Eq. (42) to obtain

$$
\begin{array}{rlrl}
\chi_{p q, r s}^{0}(i \omega) & =\frac{1}{i \omega-\varepsilon_{i a}} \quad \text { for } p=s=i \text { and } q=r=a \\
& =\frac{1}{-i \omega-\varepsilon_{i a}} & \text { for } p=s=a \text { and } q=r=i \\
& =0 & & \text { else },
\end{array}
$$

as usual with $i$ denoting occupied and $a$ denoting unoccupied orbitals. Note that by $i$ in front of the frequency $\omega$ the imaginary unit not the index $i$ is denoted. By $\varepsilon_{i a}=\varepsilon_{a}-\varepsilon_{i}$ again differences of single-particle eigenvalues are denoted. With this Eq. (41) then transforms into

$$
\begin{aligned}
E_{0}= & \sum_{p q}\left[h_{p q}+\frac{1}{4} \sum_{r}\langle p r \| q r\rangle+\frac{1}{4} \sum_{r s}\langle p r \| q s\rangle\left\langle\Psi_{0}\left|a_{r}^{\dagger} a_{s}\right| \Psi_{0}\right\rangle\right]\left\langle\Psi_{0}\left|a_{p}^{\dagger} a_{q}\right| \Psi_{0}\right\rangle \\
& -\frac{1}{4} \sum_{i a}\langle i a \| i a\rangle-\frac{1}{4 \pi} \sum_{p q r s}\langle p r \| q s\rangle \int_{0}^{\infty} d \omega\left[\chi_{p q, r s}(i \omega)-\chi_{p q, r s}^{0}(i \omega)\right]
\end{aligned}
$$

where it has been used that, using Eq. (43), the frequency integration over $\chi_{p q, r s}^{0}(i \omega)$ yields $-2 \pi \delta_{i j} \delta_{a b}$ and leads to $-\frac{1}{4 \pi} \sum_{p q r s}\langle p r \| q s\rangle \int_{0}^{\infty} d \omega \chi_{p q, r s}^{0}(i \omega)=\frac{1}{4} \sum_{i a}\langle i a \| i a\rangle$. with $i, j$ denoting occupied and $a, b$ unoccupied orbitals

If one now makes the approximation that the exact one-particle density matrices are identical to the density matrices of the determinantal wave function, here a KS or HF wave function, i.e. $\left\langle\Psi_{0}\left|a_{p}^{\dagger} a_{q}\right| \Psi_{0}\right\rangle \approx\left\langle\Phi_{0}\left|a_{p}^{\dagger} a_{q}\right| \Phi_{0}\right\rangle=\delta_{p q} \delta_{q i}$, then Eq. (44) simplifies to

$$
E_{0} \approx \sum_{i} h_{i i}+\frac{1}{2} \sum_{i j}\langle i j \| i j\rangle-\frac{1}{4 \pi} \sum_{p q r s}\langle p r \| q s\rangle \int_{0}^{\infty} d \omega\left[\chi_{p q, r s}(i \omega)-\chi_{p q, r s}^{0}(i \omega)\right]
$$

For the case that $\Phi_{0}$ is the Hartree-Fock determinant the first two-terms on the right hand-side of Eq. (45) correspond just to the Hartree-Fock ground-state energy and the remainder

$$
E_{c} \approx-\frac{1}{4 \pi} \sum_{p q r s}\langle p r \| q s\rangle \int_{0}^{\infty} d \omega\left[\chi_{p q, r s}(i \omega)-\chi_{p q, r s}^{\mathrm{HF}}(i \omega)\right]
$$

with $\chi_{p q, r s}^{\mathrm{HF}}(i \omega)$ denoting the Hartree-Fock response function, i.e., Eq. (43) in terms of Hartree-Fock orbital energies, is an approximation to the correlation energy.

It is instructive to evaluate the frequency integration in Eq. (46). With Eq. (42) and the integral $\int_{0}^{\infty} d \omega \omega /\left(a^{2}+\omega^{2}\right)=\pi / 2$ we obtain for the frequency integral 
over $\chi_{p q, r s}(i \omega)$ :

$$
\begin{aligned}
& \frac{-1}{4 \pi} \sum_{p q r s}\langle p r \| q s\rangle \int_{0}^{\infty} d \omega \chi_{p q, r s}(i \omega) \\
& =\frac{-1}{4 \pi} \sum_{p q r s}\langle p r \| q s\rangle \int_{0}^{\infty} d \omega \sum_{n \neq 0}\left[\frac{\left\langle\Psi_{0}\left|\hat{a}_{p}^{\dagger} \hat{a}_{q}\right| \Psi_{n}\right\rangle\left\langle\Psi_{n}\left|\hat{a}_{r}^{\dagger} \hat{a}_{s}\right| \Psi_{0}\right\rangle}{E_{0}-E_{n}+i \omega}\right. \\
& \left.+\frac{\left\langle\Psi_{n}\left|\hat{a}_{p}^{\dagger} \hat{a}_{q}\right| \Psi_{0}\right\rangle\left\langle\Psi_{0}\left|\hat{a}_{r}^{\dagger} \hat{a}_{s}\right| \Psi_{n}\right\rangle}{E_{0}-E_{n}-i \omega}\right] \\
& =\frac{-1}{4 \pi} \sum_{p q r s}\langle p r \| q s\rangle \int_{0}^{\infty} d \omega \sum_{n \neq 0}\left[\frac{\left\langle\Psi_{0}\left|\hat{a}_{p}^{\dagger} \hat{a}_{q}\right| \Psi_{n}\right\rangle\left\langle\Psi_{n}\left|\hat{a}_{r}^{\dagger} \hat{a}_{s}\right| \Psi_{0}\right\rangle}{E_{0}-E_{n}+i \omega}\right. \\
& \left.+\frac{\left\langle\Psi_{0}\left|\hat{a}_{p}^{\dagger} \hat{a}_{q}\right| \Psi_{n}\right\rangle\left\langle\Psi_{n}\left|\hat{a}_{r}^{\dagger} \hat{a}_{s}\right| \Psi_{0}\right\rangle}{E_{0}-E_{n}-i \omega}\right] \\
& =\frac{1}{2 \pi} \sum_{p q r s}\langle p r \| q s\rangle \int_{0}^{\infty} d \omega \sum_{n \neq 0} \frac{E_{n}-E_{0}}{\left(E_{n}-E_{0}\right)^{2}+\omega^{2}}\left\langle\Psi_{0}\left|\hat{a}_{p}^{\dagger} \hat{a}_{q}\right| \Psi_{n}\right\rangle\left\langle\Psi_{n}\left|\hat{a}_{r}^{\dagger} \hat{a}_{s}\right| \Psi_{0}\right\rangle \\
& =\frac{1}{4} \sum_{p q r s}\langle p r|| q s\rangle \sum_{n \neq 0}\left\langle\Psi_{0}\left|\hat{a}_{p}^{\dagger} \hat{a}_{q}\right| \Psi_{n}\right\rangle\left\langle\Psi_{n}\left|\hat{a}_{r}^{\dagger} \hat{a}_{s}\right| \Psi_{0}\right\rangle \\
& =\frac{1}{4} \sum_{p q r s}\langle p r \| q s\rangle\left\langle\Psi_{0}\left|\hat{a}_{p}^{\dagger} \hat{a}_{q} \hat{a}_{r}^{\dagger} \hat{a}_{s}\right| \Psi_{0}\right\rangle \\
& -\frac{1}{4} \sum_{p q r s}\langle p r|| q s\rangle\left\langle\Psi_{0}\left|\hat{a}_{p}^{\dagger} \hat{a}_{q}\right| \Psi_{0}\right\rangle\left\langle\Psi_{0}\left|\hat{a}_{r}^{\dagger} \hat{a}_{s}\right| \Psi_{0}\right\rangle \\
& =\frac{1}{4} \sum_{p q r s}\langle r p|| q s\rangle\left\langle\Psi_{0}\left|\hat{a}_{p}^{\dagger} \hat{a}_{r}^{\dagger} \hat{a}_{q} \hat{a}_{s}\right| \Psi_{0}\right\rangle+\frac{1}{4} \sum_{p q s}\langle p q \| q s\rangle\left\langle\Psi_{0}\left|\hat{a}_{p}^{\dagger} \hat{a}_{s}\right| \Psi_{0}\right\rangle \\
& -\frac{1}{4} \sum_{p q r s}\langle p r|| q s\rangle\left\langle\Psi_{0}\left|\hat{a}_{p}^{\dagger} \hat{a}_{q}\right| \Psi_{0}\right\rangle\left\langle\Psi_{0}\left|\hat{a}_{r}^{\dagger} \hat{a}_{s}\right| \Psi_{0}\right\rangle \\
& =V_{e e}-\frac{1}{4} \int d \mathbf{r} d \mathbf{r}^{\prime}\left[\frac{\rho(\mathbf{r}) \rho\left(\mathbf{r}^{\prime}\right)}{\left|\mathbf{r}-\mathbf{r}^{\prime}\right|}-\frac{\rho\left(\mathbf{r}^{\prime}, \mathbf{r}\right) \rho\left(\mathbf{r}, \mathbf{r}^{\prime}\right)}{\left|\mathbf{r}-\mathbf{r}^{\prime}\right|}\right] \\
& +\frac{1}{4} \int d \mathbf{r} d \mathbf{r}^{\prime} \frac{\delta\left(\mathbf{r}-\mathbf{r}^{\prime}\right) \rho(\mathbf{r})}{\left|\mathbf{r}-\mathbf{r}^{\prime}\right|}-\frac{1}{4} \int d \mathbf{r} d \mathbf{r}^{\prime} \sum_{q} \frac{\phi_{q}^{\dagger}\left(\mathbf{r}^{\prime}\right) \phi_{q}\left(\mathbf{r}^{\prime}\right) \rho(\mathbf{r})}{\left|\mathbf{r}-\mathbf{r}^{\prime}\right|} \text {. }
\end{aligned}
$$

In Eq. (47) it was used that the summation indices can be renamed ( $r$ to $p, p$ to $r, s$ to $q, q$ to $s)$ and $\langle r p \| s q\rangle=\langle p r \| q s\rangle$, that $\hat{a}_{q} \hat{a}_{r}^{\dagger}=\delta_{q r}-\hat{a}_{r}^{\dagger} \hat{a}_{q}$, that $\langle r p \| q s\rangle=$ - $\langle p r \| q s\rangle$, that $\frac{1}{4} \sum_{p q r s}\langle r p \| q s\rangle\left\langle\Psi_{0}\left|\hat{a}_{p}^{\dagger} \hat{a}_{r}^{\dagger} \hat{a}_{q} \hat{a}_{s}\right| \Psi_{0}\right\rangle$ equals the electron-electron interaction energy $V_{e e}$ of an electronic system, that $\sum_{r s} \phi_{r}^{\dagger}(\mathbf{r}) \phi_{s}\left(\mathbf{r}^{\prime}\right)\left\langle\Psi_{0}\left|\hat{a}_{r}^{\dagger} \hat{a}_{s}\right| \Psi_{0}\right\rangle$ equals the first order density matrix $\rho\left(\mathbf{r}, \mathbf{r}^{\prime}\right)$, and that $\sum_{q} \phi_{q}(\mathbf{r}) \phi_{q}^{\dagger}\left(\mathbf{r}^{\prime}\right)$ is a representation of the delta function $\delta\left(\mathbf{r}-\mathbf{r}^{\prime}\right)$. The last two terms in Eq. (47) are singular and thus, strictly speaking, illdefined.

The frequency integration of the terms in Eq. (46) that contain HF response 
Here $V_{e e}^{\mathrm{HF}}$ denotes the electron-electron interaction energy of the HF determinant and $\rho^{\mathrm{HF}}\left(\mathbf{r}, \mathbf{r}^{\prime}\right)$ and $\rho^{\mathrm{HF}}(\mathbf{r})$ are the HF first order density matrix and the HF electron density, respectively. Subtraction of Eq. (48) from Eq. (47) yields:

$$
\begin{aligned}
E_{c} \approx-\frac{1}{4 \pi} \sum_{p q r s}\langle & p r \| q s\rangle \int_{0}^{\infty} d \omega\left[\chi_{p q, r s}(i \omega)-\chi_{p q, r s}^{\mathrm{HF}}(i \omega)\right] \\
= & V_{e e}-V_{e e}^{\mathrm{HF}}-\frac{1}{4} \int d \mathbf{r} d \mathbf{r}^{\prime}\left[\frac{\rho(\mathbf{r}) \rho\left(\mathbf{r}^{\prime}\right)}{\left|\mathbf{r}-\mathbf{r}^{\prime}\right|}-\frac{\rho^{\mathrm{HF}}(\mathbf{r}) \rho^{\mathrm{HF}}\left(\mathbf{r}^{\prime}\right)}{\left|\mathbf{r}-\mathbf{r}^{\prime}\right|}\right] \\
& +\frac{1}{4} \int d \mathbf{r} d \mathbf{r}^{\prime}\left[\frac{\rho\left(\mathbf{r}^{\prime}, \mathbf{r}\right) \rho\left(\mathbf{r}, \mathbf{r}^{\prime}\right)}{\left|\mathbf{r}-\mathbf{r}^{\prime}\right|}-\frac{\rho^{\mathrm{HF}}\left(\mathbf{r}^{\prime}, \mathbf{r}\right) \rho^{\mathrm{HF}}\left(\mathbf{r}, \mathbf{r}^{\prime}\right)}{\left|\mathbf{r}-\mathbf{r}^{\prime}\right|}\right] \\
& +\frac{1}{4} \int d \mathbf{r} d \mathbf{r}^{\prime} \frac{\delta\left(\mathbf{r}-\mathbf{r}^{\prime}\right) \rho(\mathbf{r})}{\left|\mathbf{r}-\mathbf{r}^{\prime}\right|}-\frac{1}{4} \int d \mathbf{r} d \mathbf{r}^{\prime} \frac{\delta\left(\mathbf{r}-\mathbf{r}^{\prime}\right) \rho^{\mathrm{HF}}(\mathbf{r})}{\left|\mathbf{r}-\mathbf{r}^{\prime}\right|} \\
& -\frac{1}{4} \int d \mathbf{r} d \mathbf{r}^{\prime} \sum_{q} \frac{\phi_{q}^{\dagger}\left(\mathbf{r}^{\prime}\right) \phi_{q}\left(\mathbf{r}^{\prime}\right) \rho(\mathbf{r})}{\left|\mathbf{r}-\mathbf{r}^{\prime}\right|}+\frac{1}{4} \int d \mathbf{r} d \mathbf{r}^{\prime} \sum_{q} \frac{\phi_{q}^{\dagger}\left(\mathbf{r}^{\prime}\right) \phi_{q}\left(\mathbf{r}^{\prime}\right) \rho^{\mathrm{HF}}(\mathbf{r})}{\left|\mathbf{r}-\mathbf{r}^{\prime}\right|}
\end{aligned}
$$

The right hand side of Eq. (49) contains the electron-electron interaction contribution to the correlation energy, i.e., the difference $V_{e e}-V_{e e}^{\mathrm{HF}}$, plus various difference terms containing the exact and the $\mathrm{HF}$ first order density matrix and electron density. In order to associate the right hand side of Eq. (49) with the correlation energy as it is done within the RPA, Eq. (46), the approximation that the HF first order density matrix equals the exact first order density matrix has to be made as has been used above. With this approximation, which implies the equality of the HF and the exact electron density, all the difference terms on the right hand side of Eq. (49) cancel. Moreover, under this approximation there would be no kinetic contribution to the correlation energy and no contribution from the electron nuclei interaction and therefore there would remain only the electron-electron interaction contribution $V_{e e}-V_{e e}^{\mathrm{HF}}$ to the correlation energy. This indicates that the approximation that the HF and the exact first order density matrices are equal is a quite severe one that could introduce errors of a magnitude comparable to the electron-nuclei interaction contribution and the kinetic contribution to the correlation energy. Here a marked difference to the RPA correlation energy within the KS formalism given by the adiabatic-connection dissipation-fluctuation theorem (32) 
shows up. The adiabatic-connection dissipation-fluctuation theorem (32) is exact, it yields the exact correlation energy as defined in the KS formalism. Approximations are introduced with the approximate density-functionals, the exchange-correlation potential and kernel when applying the adiabatic-connection dissipation-fluctuation theorem. In HF based RPA, with Eq. (46), right from the start approximations are introduced. It should be noted, however, that the form of the correlation energy of Eq. (49) might be even more inaccurate if KS orbitals and the corresponding $\mathrm{KS}$ response function are used instead of $\mathrm{HF}$ orbitals and the HF reponse function because the KS one-particle density matrix is neither identical to the exact density matrix nor can it be expected to be a good approximation to it, even if the exact exchange-correlation potential would have been employed, see Ref. [85], p.47 ff.

If in Eq. (49) the antisymmetrized two-electron integral $\langle p r \| q s\rangle$ is replaced by the simple nonantisymmetrized integral $\langle p r \mid q s\rangle$ and the prefactor $1 / 4 \pi$ is replaced by $1 / 2 \pi$ then carrying out the frequency integrations in the same way as before leads to

$$
\begin{aligned}
E_{c} \approx-\frac{1}{2 \pi} \sum_{p q r s} & \langle p r \mid q s\rangle \int_{0}^{\infty} d \omega\left[\chi_{p q, r s}(i \omega)-\chi_{p q, r s}^{\mathrm{HF}}(i \omega)\right] \\
= & V_{e e}-V_{e e}^{\mathrm{HF}}-\frac{1}{2} \int d \mathbf{r} d \mathbf{r}^{\prime}\left[\frac{\rho(\mathbf{r}) \rho\left(\mathbf{r}^{\prime}\right)}{\left|\mathbf{r}-\mathbf{r}^{\prime}\right|}-\frac{\rho^{\mathrm{HF}}(\mathbf{r}) \rho^{\mathrm{HF}}\left(\mathbf{r}^{\prime}\right)}{\left|\mathbf{r}-\mathbf{r}^{\prime}\right|}\right] \\
& +\frac{1}{2} \int d \mathbf{r} d \mathbf{r}^{\prime} \frac{\delta\left(\mathbf{r}-\mathbf{r}^{\prime}\right) \rho(\mathbf{r})}{\left|\mathbf{r}-\mathbf{r}^{\prime}\right|}-\frac{1}{2} \int d \mathbf{r} d \mathbf{r}^{\prime} \frac{\delta\left(\mathbf{r}-\mathbf{r}^{\prime}\right) \rho^{\mathrm{HF}}(\mathbf{r})}{\left|\mathbf{r}-\mathbf{r}^{\prime}\right|}
\end{aligned}
$$

With the assumption that the $\mathrm{HF}$ and the exact electron densities are equal the difference terms in Eq. (50) that contain the exact and the HF density vanish and the electron-electron interation contribution $V_{e e}-V_{e e}^{\mathrm{HF}}$ to the correlation energy remains. The assumption of an equality of the $\mathrm{HF}$ and the exact electron densities is weaker than the assumption of the equality of the corresponding first order density matrices. That is, Eq. (50) compared to Eq. (49) requires a weaker approximation to yield a meaningful quantity, $V_{e e}-V_{e e}^{\mathrm{HF}}$ namely. If the stronger assumption of an equality of the HF and the exact first order density matrices is made then, as before, $V_{e e}-V_{e e}^{\mathrm{HF}}$ can be associated with the complete correlation energy. Eq. (50) therefore is an alternative to Eq. (49).

Alternative to the use of Kohn-Sham orbitals or Hartree-Fock orbitals Eq. (41) could also be written in terms of natural orbitals or Brueckner orbitals. For natural orbitals the transformation from Eq. (44) to Eq. (45) holds true exactly, provided the summations run over all orbitals with nonzero occupation numbers and Eq. (43) is generalized accordingly. Brueckner orbitals, on the other hand, differ from natural orbitals in third order of perturbation theory [86]. Since it has been found that the Brueckner reference determinant of a Brueckner coupled-cluster doubles (BCCD) wave function better approximates the electron density of the unrelaxed full BCCD wave function $[87,88]$ it can be expected that the correlation energy expressions in Eqns. (46) and (50) are better approximated in terms of Brueckner instead of Hartree-Fock orbitals.

\subsection{Overview of RPA methods including exchange interactions}

In a diagrammatical perturbation expansion of the direct RPA (dRPA) correlation energy it can be seen that only certain types of diagrams occur, namely those 
that have a ring form $[9,56,89]$. Because of this the direct RPA is sometimes also termed ring approximation or direct ring coupled-cluster doubles method (drCCD). Moreover, it has been shown that the dispersion interaction energy on the coupledcluster doubles level is identical to the dispersion energy described by RPA response propagators of the monomers, see Ref. [90].

A simple Hartree calculation suffers from the neglect of electron exchange effects that leads to erroneous self-interactions [56]. This shortcoming is rectified by taking into account the exchange contributions in the Hartree-Fock method. Similarly, dRPA suffers from the neglect of exchange, now in higher orders in the electron-electron interaction. As a result, dRPA methods usually strongly overestimate electron correlation due to self-correlation errors. Most striking is the fact, that dRPA yields non-zero correlation energies in one-electron systems [37].

Because of this, self-correlation in dRPA has to be corrected by including electron exchange effects. There is, however, by no means a unique way to do this. Quite some RPA methods including exchange effects were developed over the years [13, 14, 41, 84, 91-93]. As the standard RPA or 'normal RPA' (NRPA) [92, 93] one may define the variant in which all ring-diagrams in dRPA are supplemented by additional ring-diagrams with the interaction lines replaced by antisymmetrised interaction lines. Such an RPA method sometimes is also refered to as ring coupled-cluster doubles (rCCD) approach [94].

The starting point of the various HF based RPA variants is the time-dependent HF equation [19, 93, 95-97]

$$
\left(\begin{array}{cc}
\varepsilon+\mathbf{A} & \mathbf{B} \\
-\mathbf{B} & -\varepsilon-\mathbf{A}
\end{array}\right)\left(\begin{array}{ll}
\mathbf{X} & \mathbf{Y} \\
\mathbf{Y} & \mathbf{X}
\end{array}\right)=\left(\begin{array}{ll}
\mathbf{X} & \mathbf{Y} \\
\mathbf{Y} & \mathbf{X}
\end{array}\right)\left(\begin{array}{cc}
\boldsymbol{\Omega} & 0 \\
\mathbf{0} & -\boldsymbol{\Omega}
\end{array}\right)
$$

whose solutions and eigenvalues are used to construct an approximation to the response matrix elements $\chi_{p q, r s}(i \omega)$ required in the basic equation (46). The matrices $\varepsilon, \mathbf{A}, \mathbf{B}, \mathbf{X}, \mathbf{Y}$, and $\boldsymbol{\Omega}$ have the dimension of the number of products of occupied and unoccupied spin orbitals. The matrix elements are defined anlogously as in the KS case, however, with respect to HF spin orbitals instead of KS spatial orbitals, i.e., $\varepsilon_{i a, j b}=\delta_{i j} \delta_{a b}\left(\varepsilon_{i}-\varepsilon_{a}\right), A_{i a, j b}=\langle i j \mid a b\rangle-\langle i a \mid j b\rangle$, and $B_{i a, j b}=\langle i j \mid a b\rangle-\langle i j \mid b a\rangle$. Again orbitals and subsequently all occuring matrices are assumed to be real-valued. In a non-spin-polarized electron system the time dependent HF equation (51) can be decoupled in equations for singlet-singlet and singlet-triplet excitations with matrices $\boldsymbol{\varepsilon}, \mathbf{A}, \mathbf{B}, \mathbf{X}$ and $\mathbf{Y}$ that have the dimension of the number products of occupied and unoccupied spatial orbitals only, i.e., a dimension that is a factor of four smaller than in the general spin-polarized case. Note that Eq. (51) has pairs of solutions with eigenvalues $\Omega_{n}$ and $-\Omega_{n}$. The eigenvalues $\Omega_{n}$ are the excitation energies within the RPA.

The eigenvectors given by the matrices $\mathbf{X}$ and $\mathbf{Y}$ fulfill the normalisation condition [19]

$$
\left(\begin{array}{ll}
\mathbf{X}^{T} & \mathbf{Y}^{T} \\
\mathbf{Y}^{T} & \mathbf{X}^{T}
\end{array}\right)\left(\begin{array}{cc}
\mathbf{1} & \mathbf{0} \\
\mathbf{0} & -\mathbf{1}
\end{array}\right)\left(\begin{array}{ll}
\mathbf{X} & \mathbf{Y} \\
\mathbf{Y} & \mathbf{X}
\end{array}\right)=\left(\begin{array}{cc}
\mathbf{1} & \mathbf{0} \\
\mathbf{0} & -\mathbf{1}
\end{array}\right)
$$

Once Eq. (51) has been solved, the RPA response matrix $\chi^{\mathrm{RPA}}(\nu)$ can be expressed in the spectral form

$$
\chi^{\mathrm{RPA}}(\nu)=\left(\begin{array}{ll}
\mathbf{X} & \mathbf{Y} \\
\mathbf{Y} & \mathbf{X}
\end{array}\right)\left(\begin{array}{cc}
(\nu \mathbf{1}-\boldsymbol{\Omega})^{-1} & \mathbf{0} \\
\mathbf{0} & (-\nu \mathbf{1}-\boldsymbol{\Omega})^{-1}
\end{array}\right)\left(\begin{array}{ll}
\mathbf{X}^{T} & \mathbf{Y}^{T} \\
\mathbf{Y}^{T} & \mathbf{X}^{T}
\end{array}\right)
$$


The elements of $\chi^{\mathrm{RPA}}(\nu)$ with $\nu=i \omega$ enter as approximation of the exact response matrix elements $\chi_{p q, r s}(i \omega)$ Eq. (46) for the RPA correlation energy, leading to

$$
E_{c}^{\mathrm{RPA}}=\frac{-1}{4 \pi} \sum_{p q r s} \int_{0}^{\infty} d \omega \operatorname{Tr}\left[\mathbf{W}^{\mathrm{RPA}}\left[\chi^{\mathrm{RPA}}(i \omega)-\chi^{\mathrm{HF}}(i \omega)\right]\right]
$$

with the $\mathrm{HF}$ response matrix $\chi^{\mathrm{HF}}(\nu)$ containing elements given in Eq. (43) and with the interaction matrix $\mathbf{W}^{\mathrm{RPA}}$ defined as

$$
\mathbf{W}^{\mathrm{RPA}}=\left(\begin{array}{ll}
\mathbf{A} & \mathbf{B} \\
\mathbf{B} & \mathbf{A}
\end{array}\right)
$$

Alternatively the RPA response function can be obtained from the Dyson-like equation

$$
\chi^{\mathrm{RPA}}(\nu)=\chi^{\mathrm{HF}}(\nu)+\chi^{\mathrm{HF}}(\nu) \mathbf{W}^{\mathrm{RPA}} \chi^{\mathrm{RPA}}(\nu)
$$

Repeated insertion of Eq. (55) into itself leads to a series expansion of $\chi^{\mathrm{RPA}}(\nu)$. If this expansion is used to evaluate the RPA correlation energy $E_{c}^{\mathrm{RPA}}$, also the latter is obtained in a series expansion. Comparing this expansion of $E_{c}^{\mathrm{RPA}}$ with manybody perturbation theory shows that the expansion of Eq. (55) produces wrong prefactors in front of all terms. It has been shown that this can be remedied through the introduction of an interaction strength parameter $\alpha$ in Eq. (46) $[6,98,99]$ which then in the RPA approach is written as:

$$
\begin{aligned}
E_{c}^{\mathrm{RPA}} & =-\frac{1}{4 \pi} \sum_{p q r s}\langle p r \| q s\rangle \int_{0}^{1} d \alpha \int_{0}^{\infty} d \omega\left[\chi_{p q, r s}^{\mathrm{RPA}}(i \omega, \alpha)-\chi_{p q, r s}^{\mathrm{HF}}(i \omega)\right] \\
& =\frac{1}{2} \operatorname{Tr}\left(\mathbf{W}^{\mathrm{RPA}} \mathbf{P}\right)
\end{aligned}
$$

where $\mathbf{P}=-(1 / 2 \pi) \int_{0}^{1} d \alpha \int_{0}^{\infty} d \omega\left[\chi^{\mathrm{RPA}}(i \omega, \alpha)-\chi^{\mathrm{HF}}(i \omega)\right]$ denotes the correlation part of the pair density. The matrix $\chi^{\mathrm{RPA}}(i \omega, \alpha)$ is obtained by scaling the matrices $\mathbf{A}$ and $\mathbf{B}$ in Eq. (51) or the matrix $\mathbf{W}$ in Eq. (55) by the coupling constant $\alpha$. Since $\mathbf{W}$ is proportional to the electronic interaction, the RPA response function can then be expanded in a power series of $\alpha$ via the Dyson Eq. (55). A very crucial point is now, that in the standard RPA method the interaction strength integration is not performed exactly. Instead one assumes that the leading order term $\boldsymbol{\chi}_{0} \mathbf{W}^{\mathrm{RPA}}(\alpha) \boldsymbol{\chi}_{0}$ of the Dyson type Eq. (55) that is linear in $\alpha$ is dominating the perturbation expansion and sets:

$$
\int_{0}^{1} d \alpha \chi^{\mathrm{RPA}}(\nu, \alpha) \approx \frac{1}{2} \chi^{\mathrm{RPA}}(\nu, \alpha=1)
$$

which corresponds to a coupling strength average. With this the correlation energy gets

$$
E_{c}^{\mathrm{RPA}} \approx-\frac{1}{8 \pi} \sum_{p q r s}\langle p r \| q s\rangle \int_{0}^{\infty} d \omega\left[\chi_{p q, r s}^{\mathrm{RPA}}(i \omega, \alpha=1)-\chi_{p q, r s}^{\mathrm{HF}}(i \omega)\right]
$$

and the insertion of the spectral representation of the response function (Eq. (53)) 
yields after integration over $\omega$

$$
\begin{aligned}
E_{c}^{\mathrm{RPA}} & =\frac{1}{8} \operatorname{Tr}\left\{\left(\begin{array}{ll}
\mathbf{A} & \mathbf{B} \\
\mathbf{B} & \mathbf{A}
\end{array}\right) \circ\left[\left(\begin{array}{ll}
\mathbf{X} \mathbf{X}^{T} & \mathbf{X} \mathbf{Y}^{T} \\
\mathbf{Y} \mathbf{X}^{T} & \mathbf{Y Y} \mathbf{Y}^{T}
\end{array}\right)-\left(\begin{array}{ll}
\mathbf{1} & \mathbf{0} \\
\mathbf{0} & \mathbf{1}
\end{array}\right)\right]\right\} \\
& =\frac{1}{4}\left[\operatorname{Tr}\left(\mathbf{B} \mathbf{Y} \mathbf{X}^{T}\right)+\operatorname{Tr}\left(\mathbf{A Y Y} \mathbf{Y}^{T}\right)\right]
\end{aligned}
$$

where the operator $\circ$ here defines an elementwise matrix multiplication and the normalisation condition Eq. (52) has been employed. The second term in Eq. (59) is small compared to the first term since it is of second order in the small eigenvector components $\mathbf{Y}$. Thus

$$
E_{c}^{\mathrm{RPA}} \cong \frac{1}{4} \operatorname{Tr}\left(\mathbf{B Y} \mathbf{X}^{T}\right)
$$

Using the normalisation condition $\mathbf{X}^{T} \mathbf{X}-\mathbf{Y}^{T} \mathbf{Y}=\mathbf{1}$ (see Eq. (52)) it is easy to show that $\mathbf{Y} \mathbf{X}^{T} \mathbf{X}=\mathbf{Y}+\mathbf{Y} \mathbf{Y}^{T} \mathbf{Y}$ and thus $[18,100]$

$$
\mathbf{Y X}^{T}=\mathbf{Y} \mathbf{X}^{-1}+\mathbf{Y} \mathbf{Y}^{T} \mathbf{Y} \mathbf{X}^{-1}=\left(\mathbf{1}+\mathbf{Y Y}^{T}\right) \mathbf{Y X}^{-1} \approx \mathbf{Y X}^{-1}
$$

where again it has been made use of the fact that $\mathbf{Y} \mathbf{Y}^{T} \ll \mathbf{1}$. With Eq. (61) the RPA correlation energy in Eq. (60) then can be written as

$$
E_{c}^{\mathrm{RPA}} \cong \frac{1}{4} \operatorname{Tr}\left(\mathbf{B Y X}^{-1}\right)
$$

This is the RPA correlation energy expression which is identical to the ringapproximation in coupled-cluster doubles theory (rCCD) as recently was shown by Scuseria [94]. The amplitudes defined as $\mathbf{T}=\mathbf{Y X}^{-1}$ can alternatively to the solution of Eq. (51) be obtained from the solution of the Riccati equation [101]

$$
\mathbf{B}+\mathbf{T} \varepsilon+\varepsilon \mathbf{T}+\mathbf{T A}+\mathbf{A T}+\mathbf{T B T}=\mathbf{0}
$$

and it can be shown that the RPA correlation energy defined in Eq. (62) can be calculated in terms of a sum over the differences of the RPA and SCI (singles configuration interaction) respectively (TDA) Tamm-Dancoff approximation excitation energies:

$$
E_{c}^{\mathrm{RPA}}=\frac{1}{4} \sum_{n} \Omega_{n}^{\mathrm{RPA}}-\frac{1}{4} \operatorname{Tr}(\boldsymbol{\varepsilon}+\mathbf{A})=\frac{1}{4} \sum_{n}\left[\Omega_{n}^{\mathrm{RPA}}-\Omega_{n}^{\mathrm{TDA}}\right]
$$

where it was used that the trace of a matrix does not change under cyclic permutations. Eq. (64) is the plasmon formula expression which has been obtained by McLachlan and Ball $[13,14]$ who assumed that the RPA excitations should be treated as a set of harmonic oscillators and that Eq. (64) is the zero-point energy of these oscillators. Eqs. (62) and (64) are given in the spin-orbital basis. In the spatial orbital basis the expressions transform into [84, 93] 


$$
\begin{aligned}
E_{c}^{\mathrm{RPA}} & =\frac{1}{4}\left[\operatorname{Tr}\left({ }^{1} \mathbf{B}^{1} \mathbf{T}\right)+3 \operatorname{Tr}\left({ }^{3} \mathbf{B}^{3} \mathbf{T}\right)\right] \\
& =\frac{1}{4} \sum_{i} \sum_{S=0}^{1}(2 S+1)\left[\omega_{i}^{\mathrm{RPA}}(S)-\omega_{i}^{\mathrm{TDA}}(S)\right]
\end{aligned}
$$

where $S=0,1$ refer to singlet and triplet excitation energies, respectively (Eq. (51) yields $n_{\text {occ }} \times n_{\text {virt }}$ singlet excitations and $3 \times n_{\text {occ }} \times n_{\text {virt }}$ triplet excitations which appear in sets with three-fold degeneracy). The matrices ${ }^{1} \mathbf{B}$ and ${ }^{3} \mathbf{B}$ correspond to antisymmetrised integrals for the singlet and triplet case with ${ }^{1} B_{\mathrm{ia}, \mathrm{jb}}=\langle\mathrm{ij} \mid \mathrm{ab}\rangle-\langle\mathrm{ij} \mid \mathrm{ba}\rangle$ and ${ }^{3} B_{\mathrm{ia}, \mathrm{jb}}=-\langle\mathrm{ij} \mid \mathrm{ba}\rangle$ with $\mathrm{i}, \mathrm{j}, \ldots$ refering to occupied and $a, b, \ldots$ to unoccupied spatial orbitals.

It should now be noted that the expression in Eqs. (64) and (65) for the RPA correlation energy is not the only possibility to describe electron correlation on the RPA level. First of all, as explained above, Eq. (62) is only an approximation to Eq. (59). In order to distinguish between the different approaches that will be described below, we will term the approximation according to Eq. (62) rCCD-RPA or 'normal RPA one' (NRPA1) as suggested by Szabo and Ostlund [93] (to distinguish it from self-consistent RPA schemes described below). The factor of $1 / 2$ from the coupling strength average (Eq. (57)) in Eq. (64) makes the NRPA1 correlation energy exact to second order of perturbation theory. Another proposal for obtaining an energy expression that is exact to second order has been given by Fukuda [91] who omits the coupling strength integration and subtracts the correlation energy at second order from the corresponding homogeneous electron gas correlation energy. His expression is thus given by

$$
E_{c}^{\mathrm{NRPA} 2}=2 E_{c}^{\mathrm{NRPA} 1}-E_{c}^{(2)}
$$

with $E_{c}^{(2)}$ being identical to the second-order Møller-Plesset (MP2) correlation energy if a Hartree-Fock reference determinant is used. We call this approach NRPA2.

The motivation for the third variant is related to an inconsistency in the RPA twoparticle density matrix in the Hartree-Fock orbital basis set. It should hold that the singlet amplitudes ${ }^{1} \mathbf{Y}^{1} \mathbf{X}^{T}$ must be equal to the negative triplet amplitudes $-{ }^{3} \mathbf{Y}^{3} \mathbf{X}^{T}$ (see, e.g., table I in Ref. [84]). This requirement stems from the condition that the wave function is an eigenfunction of the square of the spin operator with eigenvalue zero. As a matter of fact, this condition is not fulfilled if the HartreeFock determinant is used to build the RPA response function [14, 16, 84]. The dilemma within the TDHF method is, that the linear combinations of single-excited states are produced in two ways: (1) by exciting from the HF ground-state or (2) by de-exciting from a doubly excited state of the true ground-state [21]. Since, however, the HF wave function approximates the ground-state wave function in TDHF, the de-excitation violates the Pauli exclusion principle and thus there exists an incompatibility between the wave function and the excitation operator in TDHF. Indeed, the HF ground state is often triplet unstable or yields very poor triplet excitation energies as compared to its singlet excitations [21-25]. In order to remedy this deficiency higher order RPA (HRPA) methods have been propsed in which the RPA ground state is the sum of the Hartree-Fock ground state and doubly excited 
states [16, 84, 92, 102-108]:

$$
\Psi_{0}^{\mathrm{RPA}}=\left[1+\sum_{i a, j b} T_{i j}^{a b} a_{a i}^{\dagger} a_{b j}^{\dagger}\right] \Phi_{0}^{\mathrm{HF}}
$$

The HRPA correlation coefficients $T_{i j}^{a b}$ are then determined by iteration. In the first cycle the generalised RPA eigenvalue equation

$$
\left(\begin{array}{cc}
\varepsilon+\mathcal{A} & \mathcal{B} \\
-\mathcal{B} & -\varepsilon-\mathcal{A}
\end{array}\right)\left(\begin{array}{ll}
\mathbf{X} & \mathbf{Y} \\
\mathbf{Y} & \mathbf{X}
\end{array}\right)=\left(\begin{array}{cc}
\mathbf{S} & 0 \\
\mathbf{0} & -\mathbf{S}
\end{array}\right)\left(\begin{array}{ll}
\mathbf{X} & \mathbf{Y} \\
\mathbf{Y} & \mathbf{X}
\end{array}\right)\left(\begin{array}{ll}
\Omega & 0 \\
\mathbf{0} & \Omega
\end{array}\right)
$$

is solved using the approximation $\Psi_{0}^{\mathrm{RPA}} \approx \Phi_{0}^{\mathrm{HF}}$ with $(\varepsilon+\mathcal{A})_{i a, j b}=\left\langle\Psi_{i}^{a}\left|\hat{H}-E_{0}\right| \Psi_{j}^{b}\right\rangle$, $\mathcal{B}_{i a, j b}=\left\langle\Psi_{i j}^{a b}\left|\hat{H}-E_{0}\right| \Psi_{0}\right\rangle$ and $S_{i a, j b}=\left\langle\Psi_{i}^{a} \mid \Psi_{j}^{b}\right\rangle$ where $\hat{H}$ denotes the electronic Hamiltonian, $E_{0}$ is the Hartree-Fock ground-state energy and $\Psi_{i}^{a}$ and $\Psi_{i j}^{a b}$ denote singly and doubly excited wave functions, respectively. Note that for the case $\Psi_{0}^{\mathrm{RPA}} \approx \Phi_{0}^{\mathrm{HF}}$ Eq. (68) reduces to Eq. (51) and the NRPA1 correlation energy results as first estimate. Then the correlation coefficients are used to construct the RPA wave function in Eq. (67) which in turn is used to construct the Hessian matrix in Eq. (68) to obtain new amplitudes and so forth. It should be noted that depending on which type of amplitudes are iterated different HRPA schemes will arise. While Shibuya and McKoy iterate on the $\mathbf{T}=\mathbf{Y X}^{-1}$ [102-104] Oddershede and Jørgensen [16, 18, 24, 84] use the amplitudes $\mathbf{T}=\mathbf{Y X}^{T}$ which, as described above, differ from $\mathbf{T}=\mathbf{Y X}^{-1}$ in third order of $\mathbf{Y}$. Other related methods are the self-consistent polarisation propagator approximation (SPPA) [16, 105] and the second-order polarisation propagator approximation (SOPPA) [84, 108] which iterate on the two-particle density matrix until a self-consistency is achieved. All these approaches lead to considerable improvements over TDHF (RPA in the HF basis) for the description of triplet excited states [84]. For example in SOPPA the spin-symmetry conditions of the two-particle density matrix are fulfilled [84]. Also, if the matrices $\mathcal{A}$ in Eq. (68) are augmented with two-particle two-hole corrections [109-111] such higher order RPA methods will become exact in third-order of perturbation theory which is not true for any normal random-phase approximation approach, see below and Ref. [112].

Here we will not discuss the higher RPA approaches further, but we may assume that the condition ${ }^{1} \mathbf{Y}^{1} \mathbf{X}^{T}=-{ }^{3} \mathbf{Y}^{3} \mathbf{X}^{T}=$ holds true and rewrite Eq. (62) solely in terms of the singlet amplitudes to obtain

$$
E_{c}^{\mathrm{NRPA} 3}=\frac{1}{2} \operatorname{Tr}\left(\left[{ }^{1} \mathbf{B}-{ }^{3} \mathbf{B}\right]{ }^{1} \mathbf{Y}^{1} \mathbf{X}^{-1}\right)
$$

This expression, which we term here as NRPA3 correlation energy, has been given by Szabo and Ostlund [93] and also by Oddershede [84]. As in the case of the NRPA1 and NRPA2 approches (Eqs. (65) and (66)) it is exact to second-order of perturbation theory but has the advantage that it can not be affected by triplet instabilities, since the triplet amplitudes do not enter Eq. (69). However, it has been shown by Szabo and Ostlund [93] that neither of the three approaches have the desirable property that they describe the long-range interaction energy between two molecules on the coupled Hartree-Fock level. In fact they contain erroneous terms that behave as $R^{-7}$ with the distance $R$ of the monomers and thus can not be expected to yield accurate intermolecular interaction energies [93]. As shown by Szabo and Ostlund [93, 113], an RPA method which has the correct long-range behaviour, i.e., which describes dispersion interactions on the coupled HF level is 


\section{Molecular Physics}

given by the following expression for the correlation energy:

$$
E_{c}^{\mathrm{NRPA} 4}=\frac{1}{2} \operatorname{Tr}\left({ }^{1} \mathbf{B}\left[{ }^{1} \mathbf{Y}^{1} \mathbf{X}^{-1}+{ }^{3} \mathbf{Y}^{3} \mathbf{X}^{-1}\right]\right)
$$

There also exist RPA approaches which do not explicitly account for electron exchange in the construction of the response function but add exchange effects to the direct RPA such that exactness in second order is fulfilled. Note that the dRPA approach corresponds to the approximation $\mathbf{A} \approx \mathbf{C}$ and $\mathbf{B} \approx \mathbf{C}$ in the RPA eigenvalue equation Eq. (51) with $C_{i a, j b}=\langle i j \mid a b\rangle$. The first variant is the RPA+SOX method where the exchange contribution in second order given by [38]

$$
E_{c, \text { exchange }}^{(2)}=-\frac{1}{2} \sum_{i a, j b} \frac{\langle i j \mid a b\rangle\langle i j \mid b a\rangle}{\varepsilon_{i}+\varepsilon_{j}-\varepsilon_{a}-\varepsilon_{b}}
$$

is added to the dRPA correlation energy:

$$
E_{c}^{\mathrm{RPA}+\mathrm{SOX}}=E_{c}^{\mathrm{dRPA}}+E_{c, \text { exchange }}^{(2)}
$$

A variant to this method uses the corresponding second-order Epstein-Nesbet correlation correction to the RPA and is termed RPA+RSOX [38], motivated by the fact that in RPA+SOX the self-correlation is overcorrected since the Coulomb contribution in SOX is unscreened compared to the dRPA. In another approach by Kresse et al. [41] the dRPA amplitudes $\mathbf{T}^{\mathrm{dRPA}}$ are contracted with antisymmetrised two-electron integrals:

$$
E_{c}^{\mathrm{RPA}-\mathrm{SOSEX}}=\frac{1}{2} \operatorname{Tr}\left(\mathbf{B T}^{\mathrm{dRPA}}\right)
$$

This method is termed RPA with second-order screened exchange and has the big advantage that it can efficiently be implemented in plane-wave basis function programs for solids [41].

None of the RPA methods described in this section so far carries out a coupling strength integration like the methods for the correlation energy derived from the AC-FDT formula, see sections 2.4 and 3.1. While it has been shown in section 3.1 that the AC-FDT is not rigorously defined for Hartree-Fock reference determinants, it nonetheless has the advantage that, as the NRPA4 method, it describes the longrange correlation energy between two molecules on the coupled Hartree-Fock level. It has been argued above that Eq. (46) can be written as

$$
\begin{aligned}
E_{c} & =-\frac{1}{2 \pi} \sum_{p q r s}\langle p r \mid q s\rangle \int_{0}^{\infty}\left[\chi_{p q, r s}(i \omega)-\chi_{p q, r s}^{\mathrm{HF}}(i \omega)\right] d \omega \\
& =\frac{1}{2} \operatorname{Tr}(\widehat{\mathbf{W}} \mathbf{P})
\end{aligned}
$$

by using the inherent antisymmetry property of the two-particle density matrix. The term $\widehat{\mathbf{W}}$ in Eq. (74) here defines the interaction matrix containing only nonantisymmetrised integrals. Since now again, within the RPA this equation yields wrong prefactors in the perturbation expansion tems, we introduce an additional integration over the coupling strength to obtain the correlation energy within the 
adiabatic-connection fluctuation-dissipation theorem

$$
E_{c}^{\mathrm{AC}-\mathrm{FDT}}=-\frac{1}{2 \pi} \sum_{p q r s}\langle p r \mid q s\rangle \int_{0}^{1} d \alpha \int_{0}^{\infty}\left[\chi_{p q, r s}(i \omega, \alpha)-\chi_{p q, r s}^{\mathrm{HF}}(i \omega)\right] d \omega
$$

which corresponds to Eq. (50) and defines the correlation energy in terms of the coupled and uncoupled response functions $\chi$ and $\chi^{\mathrm{HF}}$. If here $\chi$ is approximated as $\chi \approx \chi^{\mathrm{RPA}}$ one obtains the adiabatic connection RPA method (AC-RPA) which can be written as $[53,114]$

$$
E_{c}^{\mathrm{AC}-\mathrm{RPA}}=\frac{1}{2} \int_{0}^{1} d \alpha \operatorname{Tr}\left[\mathbf{C}\left[\left(\mathbf{X}_{\alpha}+\mathbf{Y}_{\alpha}\right)\left(\mathbf{X}_{\alpha}+\mathbf{Y}_{\alpha}\right)^{T}-\mathbf{1}\right]\right]=\frac{1}{2} \int_{0}^{1} d \alpha \operatorname{Tr}\left[\mathbf{C} \mathbf{P}_{\alpha}\right]
$$

where the matrix $\mathbf{C}$ is defined by the two-electron integral matrix elements $C_{i a, j b}=$ $\langle i j \mid a b\rangle$. The coefficients $\mathbf{X}_{\alpha}$ and $\mathbf{Y}_{\alpha}$ at coupling strength $\alpha$ are obtained from the solution of the eigensystem Eq. (51) if the matrices $\mathbf{A}$ and $\mathbf{B}$ are scaled by $\alpha$. Note that only the singlet excitation vectors need to be computed for calculating the AC-RPA correlation energy of Eq. (76). In order to compare this expression with the NRPA methods we can make use of the identity $(\mathbf{X}+\mathbf{Y})(\mathbf{X}+\mathbf{Y})^{T}-\mathbf{1}=$ $2 \mathbf{Y X}^{-1}+2 \mathbf{Y Y}^{T}\left(\mathbf{1}+\mathbf{Y X}^{-1}\right)$ and find, again since $\mathbf{Y} \mathbf{Y}^{T}$ is small, that

$$
E_{c}^{\mathrm{AC}-\mathrm{RPA}} \approx \int_{0}^{1} d \alpha \operatorname{Tr}\left[\mathbf{C} \mathbf{Y}_{\alpha} \mathbf{X}_{\alpha}^{-1}\right]
$$

(Basically the same transformation has been used in the derivation of Eq. (60)). The coupling-strength average of this expression is given by

$$
E_{c}^{\mathrm{AC}-\mathrm{RPA}} \approx \frac{1}{2} \operatorname{Tr}\left[\mathbf{C Y \mathbf { X } ^ { - 1 }}\right]
$$

A comparison with expression (60) for the correlation energy shows that expression (78) differs from the former by the contribution $-\frac{1}{4} \operatorname{Tr}\left[\mathbf{C Y X} \mathbf{X}^{-1}\right]-$ $\frac{1}{4} \operatorname{Tr}\left[\mathbf{K Y X}^{-1}\right]$ with the matrix $\mathbf{K}$ defined as $K_{i a, j b}=\langle i j \mid b a\rangle$. In case of the direct adiabatic-connection RPA (AC-dRPA) in which $\mathbf{X}$ and $\mathbf{Y}$ are the eigenvector components of the dRPA eigenvalue equation it has recently been shown by Jansen et al. [114] that the expressions in Eq. (76) and Eq. (78) are exactly identical, that is, the coupling-strength average of the AC-dRPA correlation energy applied to Eq. (77) containing an approximate response function is surprisingly identical to the full coupling-strength integrated expression in Eq. (76). This holds true even though the coupling-strength integrated pair density is not in general identical to the amplitudes, i.e.

$$
\int_{0}^{1} d \alpha\left(\boldsymbol{\chi}_{\alpha}-\boldsymbol{\chi}_{0}\right)=\int_{0}^{1} d \alpha\left\{\begin{array}{ll}
\mathbf{X}_{\alpha} \mathbf{X}_{\alpha}^{T} \mathbf{X}_{\alpha} \mathbf{Y}_{\alpha}^{T} \\
\mathbf{Y}_{\alpha} \mathbf{X}_{\alpha}^{T} \mathbf{Y}_{\alpha} \mathbf{Y}_{\alpha}^{T}
\end{array}\right\}-\mathbf{1} \neq \mathbf{Y X}^{-1}
$$

Since all RPA methods of this section that include exchange effects differ in third order of perturbation theory, it is instructive to derive explicit expressions for the correlation energy up to third order in terms of molecular integrals and orbital energies. For this we first expand the response function up to second order in the intermolecular interaction using Eq. (55): 


$$
\chi_{\alpha}^{(1+2)}(i \omega)=\chi_{0}(i \omega)+\alpha \chi_{0}(i \omega) \mathbf{W} \chi_{0}(i \omega)+\alpha^{2} \chi_{0}(i \omega) \mathbf{W} \chi_{0}(i \omega) \mathbf{W} \chi_{0}(i \omega)
$$

where it is employed that the interaction operator $\mathbf{W}$ linearly depends on the interaction strength $\alpha$. The RPA correlation pair density can then generally be written as

$$
\begin{array}{r}
\mathbf{P}^{(1+2)}=\int_{0}^{1} d \alpha \frac{1}{2 \pi} \int_{0}^{\infty} d \omega\left(\boldsymbol{\chi}_{\alpha}^{(2)}(i \omega)-\boldsymbol{\chi}_{0}(i \omega)\right) \\
=\int_{0}^{1} d \alpha \frac{1}{2 \pi} \int_{0}^{\infty} d \omega\left(\alpha \boldsymbol{\chi}_{0}(i \omega) \mathbf{W} \boldsymbol{\chi}_{0}(i \omega)\right. \\
\left.+\alpha^{2} \boldsymbol{\chi}_{0}(i \omega) \mathbf{W} \boldsymbol{\chi}_{0}(i \omega) \mathbf{W} \boldsymbol{\chi}_{0}(i \omega)\right)
\end{array}
$$

Now note that, as discussed above, in the NRPA approaches the coupling strength integration in Eq. (81) is not performed, but the average of Eq. (57) is taken. Because of this the NRPA and AC-RPA methods will obtain different prefactors in second order in $\mathbf{W}$ :

$$
\begin{aligned}
\mathbf{P}^{(1+2), \mathrm{NRPA}=}=\frac{1}{4 \pi} \int_{0}^{\infty} d \omega \chi_{0}(i \omega) \mathbf{W} \chi_{0}(i \omega) & \\
& +\frac{1}{4 \pi} \int_{0}^{\infty} d \omega \boldsymbol{\chi}_{0}(i \omega) \mathbf{W} \chi_{0}(i \omega) \mathbf{W} \chi_{0}(i \omega) \\
\mathbf{T}^{(1+2), \mathrm{AC}-\mathrm{RPA}=}=\frac{1}{4 \pi} \int_{0}^{\infty} d \omega \chi_{0}(i \omega) \mathbf{W} \chi_{0}(i \omega) & \\
& +\frac{1}{6 \pi} \int_{0}^{\infty} d \omega \chi_{0}(i \omega) \mathbf{W} \chi_{0}(i \omega) \mathbf{W} \chi_{0}(i \omega)
\end{aligned}
$$

The full response function (at $\alpha=1$ ) is given by (compare Eqs. (51) and (53)):

$$
\begin{aligned}
\chi_{\alpha=1}(i \omega) & =\left\{\left(\begin{array}{cc}
\varepsilon-i \omega & \mathbf{0} \\
\mathbf{0} & \varepsilon+i \omega
\end{array}\right)+\left(\begin{array}{ll}
\mathbf{A} & \mathbf{B} \\
\mathbf{B} & \mathbf{A}
\end{array}\right)\right\}^{-1} \\
& =\left[\boldsymbol{\chi}_{0}^{-1}+\mathbf{W}\right]^{-1}=\chi_{0}-\chi_{0} \mathbf{W} \chi_{0}+\chi_{0} \mathbf{W} \chi_{0} \mathbf{W} \chi_{0}+\ldots
\end{aligned}
$$

The corresponding response function for coupling strengths $\alpha$ can be obtained with Eq. (84) by scaling the interaction matrix $\mathbf{W}$ with a factor of $\alpha$. From Eq. (84) the uncoupled response function $\chi_{0}$ and the interaction operator $\mathbf{W}$ in Eqs. (82) and (83) can be identified as:

$$
\begin{aligned}
\chi_{0}(i \omega) & =\left(\begin{array}{cc}
(\varepsilon-i \omega)^{-1} & \mathbf{0} \\
\mathbf{0} & (\varepsilon+i \omega)^{-1}
\end{array}\right)=\left(\begin{array}{cc}
\frac{\varepsilon+i \omega}{\varepsilon^{2}+\omega^{2}} & \mathbf{0} \\
\mathbf{0} & \frac{\varepsilon-i \omega}{\varepsilon^{2}+\omega^{2}}
\end{array}\right)=\left(\begin{array}{cc}
\boldsymbol{\lambda}^{-} & \mathbf{0} \\
\mathbf{0} & \boldsymbol{\lambda}^{+}
\end{array}\right) \\
\mathbf{W} & =\left(\begin{array}{cc}
\mathbf{A} & \mathbf{B} \\
\mathbf{B} & \mathbf{A}
\end{array}\right)
\end{aligned}
$$


where we use the short-hand notation $\boldsymbol{\lambda}^{-}=(\varepsilon-i \omega)^{-1}$ and $\boldsymbol{\lambda}^{+}=(\varepsilon+i \omega)^{-1}$. Thus the correlated first-order RPA pair density is given by

$$
\mathbf{P}^{(1)}=\frac{1}{4 \pi} \int_{0}^{\infty} d \omega \boldsymbol{\chi}_{0}(i \omega) \mathbf{W} \boldsymbol{\chi}_{0}(i \omega)=\frac{1}{4 \pi} \operatorname{Re} \int_{0}^{\infty} d \omega\left\{\begin{array}{l}
\boldsymbol{\lambda}^{-} \mathbf{A} \boldsymbol{\lambda}^{-} \boldsymbol{\lambda}^{-} \mathbf{B} \boldsymbol{\lambda}^{+} \\
\boldsymbol{\lambda}^{+} \mathbf{B} \boldsymbol{\lambda}^{-} \boldsymbol{\lambda}^{+} \mathbf{A} \boldsymbol{\lambda}^{+}
\end{array}\right\}
$$

where it has been used that the imaginary terms of the response matrix cancel in each order of perturbation theory and thus only the real parts of the four submatrices in Eq. (87) need to be considered. Integration over $\omega$ yields:

$$
\mathbf{P}^{(1)}=\frac{1}{4}\left\{\begin{array}{cc}
\mathbf{0} & \overline{\mathbf{B}} \\
\overline{\mathbf{B}} & \mathbf{0}
\end{array}\right\}
$$

where the definition $\bar{M}_{i a, j b}=\frac{M_{i a, j b}}{\varepsilon_{i a}+\varepsilon_{j b}}$ for a matrix $\mathbf{M}$ has been used. With this result the second-order energy is given by (see Eqs. (56) and (59))

$$
\begin{array}{r}
E_{c}^{(2), \mathrm{NRPA}}=\frac{1}{4} \operatorname{Tr}\left(\mathbf{W} \mathbf{P}^{(1)}\right)=\frac{1}{8} \operatorname{Tr}\left\{\left(\begin{array}{ll}
\mathbf{A} & \mathbf{B} \\
\mathbf{B} & \mathbf{A}
\end{array}\right) \circ\left(\begin{array}{cc}
\mathbf{0} & \overline{\mathbf{B}} \\
\mathbf{B} & \mathbf{0}
\end{array}\right)\right\} \\
\frac{1}{4} \operatorname{Tr}[\mathbf{B} \overline{\mathbf{B}}]=\frac{1}{4} \sum_{i a, j b} \frac{\langle i j \| a b\rangle\langle a b \| i j\rangle}{\varepsilon_{i a}+\varepsilon_{j b}}
\end{array}
$$

where $\varepsilon_{i a}=\varepsilon_{i}-\varepsilon_{a}$. The second order energy expression in Eq. (89) can readiliy be identified as the exact second order energy if a Hartree-Fock basis is used.

Correspondingly the second-order energy of the AC-RPA method is given by

$$
\begin{array}{r}
E_{c}^{(2), \mathrm{AC}-\mathrm{RPA}}=\frac{1}{2} \operatorname{Tr}\left(\widehat{\mathbf{W}} \mathbf{P}^{(1)}\right)=\frac{1}{4} \operatorname{Tr}\left\{\left(\begin{array}{l}
\mathbf{C} \mathbf{C} \\
\mathbf{C} \mathbf{C}
\end{array}\right) \circ\left(\begin{array}{cc}
\mathbf{0} & \mathbf{B} \\
\overline{\mathbf{B}} & \mathbf{0}
\end{array}\right)\right\} \\
\frac{1}{2} \operatorname{Tr}[\mathbf{C} \overline{\mathbf{B}}]=\frac{1}{4} \sum_{i a, j b} \frac{\langle i j \| a b\rangle\langle a b \| i j\rangle}{\varepsilon_{i a}+\varepsilon_{j b}}
\end{array}
$$

and thus, too, is exact to second order of perturbation theory.

For the (unscaled, i.e. without a prefactor due to the coupling strength integration) second-order pair density one gets

$$
\begin{aligned}
\mathbf{P}^{(2)}= & \frac{1}{2 \pi} \int_{0}^{\infty} d \omega \boldsymbol{\chi}_{0}(i \omega) \mathbf{W} \boldsymbol{\chi}_{0}(i \omega) \mathbf{W} \boldsymbol{\chi}_{0}(i \omega) \\
= & \frac{1}{2 \pi} \operatorname{Re} \int_{0}^{\infty} d \omega \times \\
& \left\{\begin{array}{ll}
\boldsymbol{\lambda}^{-} \mathbf{A} \boldsymbol{\lambda}^{-} \mathbf{A} \boldsymbol{\lambda}^{-}+\boldsymbol{\lambda}^{-} \mathbf{B} \boldsymbol{\lambda}^{+} \mathbf{B} \boldsymbol{\lambda}^{-} & \boldsymbol{\lambda}^{-} \mathbf{A} \boldsymbol{\lambda}^{-} \mathbf{B} \boldsymbol{\lambda}^{+}+\boldsymbol{\lambda}^{-} \mathbf{B} \boldsymbol{\lambda}^{+} \mathbf{A} \boldsymbol{\lambda}^{+} \\
\boldsymbol{\lambda}^{+} \mathbf{B} \boldsymbol{\lambda}^{-} \mathbf{A} \boldsymbol{\lambda}^{-}+\boldsymbol{\lambda}^{+} \mathbf{A} \boldsymbol{\lambda}^{+} \mathbf{B} \boldsymbol{\lambda}^{-} & \boldsymbol{\lambda}^{+} \mathbf{B} \boldsymbol{\lambda}^{-} \mathbf{B} \boldsymbol{\lambda}^{+}+\boldsymbol{\lambda}^{+} \mathbf{A} \boldsymbol{\lambda}^{+} \mathbf{A} \boldsymbol{\lambda}^{+}
\end{array}\right\} \\
= & \frac{1}{2}\left\{\begin{array}{cc}
\widetilde{\mathbf{B B}} & \widetilde{\mathbf{A B}}+\widetilde{\mathbf{B A}} \\
\widetilde{\mathbf{A B}}+\widetilde{\mathbf{B A}} & \widetilde{\mathbf{B B}}
\end{array}\right\}
\end{aligned}
$$

where in the last expression the contraction $\widetilde{M N}_{i a, j b}=\frac{M_{i a, k c} N_{k c, j b}}{\left(\varepsilon_{i a}+\varepsilon_{j b}\right)\left(\varepsilon_{i a}+\varepsilon_{k c}\right)}$ for two 


$$
\begin{aligned}
E_{c}^{(3), \mathrm{NRPA} 1} & =\frac{1}{2} \operatorname{Tr}\left(\mathbf{W} \mathbf{P}^{(2)}\right)=\frac{1}{2} \operatorname{Tr}[\overline{\mathbf{B}} \mathbf{A} \overline{\mathbf{B}}] \\
& =\frac{1}{2} \sum_{i a, j b, k c} \frac{\langle i j \| a b\rangle\langle k b \| \mid c j\rangle\langle a c \| i k\rangle}{\left(\varepsilon_{i a}+\varepsilon_{j b}\right)\left(\varepsilon_{i a}+\varepsilon_{k c}\right)}
\end{aligned}
$$

In the AC-RPA method the pair density in Eq. (91) is integrated over the coupling-strength yielding a prefactor of $\frac{1}{3}$ (see Eq. (83)) and the result for the third order energy is (see also [114]):

$$
\begin{aligned}
E_{c}^{(3), \mathrm{AC}-\mathrm{RPA}}= & \frac{1}{2} \operatorname{Tr}\left(\widehat{\mathbf{W}} \mathbf{P}^{(2)}\right) \\
= & \frac{1}{3} \operatorname{Tr}[\overline{\mathbf{B}} \mathbf{A} \overline{\mathbf{C}}+\overline{\mathbf{A}} \mathbf{B} \overline{\mathbf{C}}+\overline{\mathbf{B}} \overline{\mathbf{B C}}] \\
= & \frac{1}{3} \sum_{i a, j b, k c}\left(\frac{\langle i j \| a b\rangle\langle j c|| b k\rangle\langle k i \mid c a\rangle}{\left(\varepsilon_{i a}+\varepsilon_{j b}\right)\left(\varepsilon_{i a}+\varepsilon_{k c}\right)}+\frac{\langle i b \| a j\rangle\langle j k \| b c\rangle\langle k i \mid c a\rangle}{\left(\varepsilon_{i a}+\varepsilon_{j b}\right)\left(\varepsilon_{i a}+\varepsilon_{k c}\right)}+\right. \\
& \left.+\frac{\langle i j \| a b\rangle\langle j k \| \mid b c\rangle\langle k c \mid i a\rangle}{\left(\varepsilon_{i a}+\varepsilon_{j b}\right)\left(\varepsilon_{i a}+\varepsilon_{k c}\right)}\right)
\end{aligned}
$$

A corresponding perturbation analysis can be made for the other RPA approaches discussed above. The result is comprised in table 2 which shows for various RPA correlation methods the prefactors of the perturbation theory terms up to third order as given in table 1.

\section{Combining elements of HF and KS based RPA}

Here we relate the EXX-RPA method of Section $2[53,55]$ to the AC-RPA method of Section 3, Eqs. (75) and (76), (termed as HF-RPA in Ref. [53]). To this end the coupling constant-dependent singlet-singlet TDHF eigenvalue equation of the AC-RPA method is written in the reduced generalised eigenvalue equation form

$$
\begin{aligned}
\left(\boldsymbol{\varepsilon}_{\mathrm{HF}}+\alpha \mathbf{A}_{\mathrm{HF}}+\alpha \mathbf{B}_{\mathrm{HF}}\right) \mathbf{u}_{n} & =\Omega_{n}^{2}\left(\boldsymbol{\varepsilon}_{\mathrm{HF}}+\alpha \mathbf{A}_{\mathrm{HF}}-\alpha \mathbf{B}_{\mathrm{HF}}\right)^{-1} \mathbf{u}_{n} \\
{\left[\boldsymbol{\varepsilon}_{\mathrm{HF}}^{2}+\alpha \varepsilon_{\mathrm{HF}}^{1 / 2}\left(\mathbf{A}_{\mathrm{HF}}+\mathbf{B}_{\mathrm{HF}}\right) \boldsymbol{\varepsilon}_{\mathrm{HF}}^{1 / 2}\right] \mathbf{z}_{n} } & =\Omega_{n}^{2}\left[\mathbf{1}+\alpha \varepsilon_{\mathrm{HF}}^{-1 / 2}\left(\mathbf{A}_{\mathrm{HF}}-\mathbf{B}_{\mathrm{HF}}\right) \boldsymbol{\varepsilon}_{\mathrm{HF}}^{-1 / 2}\right]^{-1} \mathbf{z}_{n}
\end{aligned}
$$


with $\mathbf{U}=\mathbf{X}+\mathbf{Y}$ and $\mathbf{Z}=\varepsilon_{\mathrm{HF}}^{1 / 2} \mathbf{U}$ or considering columns of the matrices $\mathbf{U}, \mathbf{X}$, $\mathbf{Y}$, an $\mathbf{Z}$, see Eq. (51), $\mathbf{u}_{n}=\mathbf{x}_{n}+\mathbf{y}_{n}$ and $\mathbf{z}_{n}=\varepsilon_{\mathrm{HF}}^{1 / 2} \mathbf{u}_{n}$. In this Section like in Section 2 non-spin-polarized electron systems are considered. The elements of the matrices $\boldsymbol{\varepsilon}_{\mathrm{HF}}, \mathbf{A}_{\mathrm{HF}}$, and $\mathbf{B}_{\mathrm{HF}}$ are defined as in the TDEXX equation (36), however, with respect to HF not EXX spatial orbitals and their eigenvalues. Because in this Section matrix elements defined with respect to $\mathrm{HF}$ or EXX orbitals occur we designate matrices with elements defined with respect to $\mathrm{HF}$ orbitals with a subscript 'HF'.

Eq. (94) can be expressed in EXX orbitals and eigenvalues if the approximation is made that the EXX orbitals and HF orbitals can be transformed into each other through an occupied-occupied virtual-virtual unitary transformation, see Refs. [70, $76,115]$ for details. This approximation is equivalent to the approximation that the EXX and the HF determinant equal each other which is known to be a very good approximation [76]. As a result of the transformation of the orbitals the matrices $\mathbf{A}_{\mathrm{HF}}$ and $\mathbf{B}_{\mathrm{HF}}$ turn into the corresponding matrices $\mathbf{A}$ and $\mathbf{B}$ with respect to KS orbitals and the matrix $\varepsilon_{\mathrm{HF}}$ turns into $\boldsymbol{\varepsilon}+\boldsymbol{\Delta}$ with $\boldsymbol{\Delta}$ defined as in the TDEXX equation (36) [69, 71]. From the transformed Eq. (94) a transformed Eq. (95) results that has the form

$$
\left[\boldsymbol{\varepsilon}^{2}+\boldsymbol{\varepsilon}^{1 / 2}[\boldsymbol{\Delta}+\alpha(\mathbf{A}+\mathbf{B})] \varepsilon^{1 / 2}\right] \mathbf{z}_{n}=\Omega_{n}^{2}\left[\mathbf{1}+\varepsilon^{-1 / 2}[\boldsymbol{\Delta}+\alpha(\mathbf{A}-\mathbf{B})] \boldsymbol{\varepsilon}^{-1 / 2}\right]^{-1} \mathbf{z}_{n}
$$

Eq. (96) would be exactly equivalent to Eq. (94) and Eq. (95) if occupied and virtual EXX and HF orbitals could be exactly transformed into each other by an occupied-occupied and a virtual-virtual unitary transformation, respectively. For two-electron systems this is indeed exactly true, for other electronic systems this is an approximation, which, however, as mentioned above, is very good and, as shown in Section 5, has only negligible effects. We therefore can consider Eq. (96) as alternative basis of the AC-RPA approach that enables a calculation of the AC-RPA correlation energy with EXX orbitals and eigenvalues and therefore lends itself to a straightforward comparison with the EXX-RPA correlation energy. The crucial difference of Eq. (96) and the TDEXX equation (36) is that the matrix $\boldsymbol{\Delta}$ in Eq. (96) is not scaled by the coupling constant because it arises there from the transformation from HF to EXX orbitals while it is scaled with the coupling constant $\alpha$ in the TDEXX equation because it emerges from the exchange kernel in this case. A second difference between Eq. (96) and Eq. (36) are the matrices on the right hand side of the equations. In Ref. [71] it was shown that the differences in the matrices on the right hand side of the equations have little effect. The reason why the EXX-RPA method yields distinctively superior results as the AC-RPA approach [55] could be attributed to the different scaling of the matrix $\boldsymbol{\Delta}$ with the coupling constant $\alpha$ [55].

We now consider a hybrid approach between the EXX-RPA and the AC-RPA method. To that end the matrix $\Delta$ in Eq. (96) is scaled by the coupling constant $\alpha$ like in the TDEXX equation (36) or conversely the form of the right hand side of the TDEXX equation (36) is changed into the form of Eq. (96) with the matrix $\Delta$ scaled by $\alpha$. This results in 


$$
\left[\varepsilon^{2}+\alpha \varepsilon^{1 / 2}(\boldsymbol{\Delta}+\mathbf{A}+\mathbf{B}) \varepsilon^{1 / 2}\right] \mathbf{z}_{n}=\Omega^{2}\left[\mathbf{1}+\alpha \varepsilon^{-1 / 2}(\boldsymbol{\Delta}+\mathbf{A}-\mathbf{B}) \varepsilon^{-1 / 2}\right]^{-1} \mathbf{z}_{n}
$$

In order to interpret the hybrid approach of Eq. (97) the matrix $\mathbf{1}+\alpha \varepsilon^{-1 / 2}(\boldsymbol{\Delta}+$ $\mathbf{A}-\mathbf{B}) \varepsilon^{-1 / 2}$ on the right hand side of Eq. (96) is expanded in a series with respect to $\alpha \varepsilon^{-1 / 2}(\boldsymbol{\Delta}+\mathbf{A}-\mathbf{B}) \varepsilon^{-1 / 2}$

$$
\begin{aligned}
& {\left[\mathbf{1}+\alpha \varepsilon^{-1 / 2}(\boldsymbol{\Delta}+\mathbf{A}-\mathbf{B}) \varepsilon^{-1 / 2}\right]^{-1}} \\
& \begin{aligned}
\boldsymbol{1} \mathbf{1}-\alpha \varepsilon^{-1 / 2}(\boldsymbol{\Delta}+\mathbf{A}-\mathbf{B}) \varepsilon^{-1 / 2} \\
\quad+\alpha^{2} \varepsilon^{-1 / 2}(\boldsymbol{\Delta}+\mathbf{A}-\mathbf{B}) \varepsilon^{-1}(\boldsymbol{\Delta}+\mathbf{A}-\mathbf{B}) \varepsilon^{-1 / 2}-\ldots
\end{aligned}
\end{aligned}
$$

If the expansion (98) is inserted into Eq. (97) and second and higher order terms are neglected then the TDEXX equation (36) results. That means the hybrid approach based on Eq. (97) differs from the EXX-RPA method only by the second and higher order contributions of the expansion (98). Inserting the expansion (98) in Eq. (97) and rearrangement suggests the eigenvalue equation

$$
\begin{aligned}
{\left[\varepsilon^{2}+\varepsilon^{1 / 2}\left[\alpha(\boldsymbol{\Delta}+\mathbf{A}+\mathbf{B})-\alpha \omega^{2}(\boldsymbol{\Delta}+\mathbf{A}-\mathbf{B})\right.\right.} & \\
& \left.\left.+\alpha^{2} \omega^{2}(\boldsymbol{\Delta}+\mathbf{A}-\mathbf{B}) \varepsilon^{-1}(\boldsymbol{\Delta}+\mathbf{A}-\mathbf{B})+\ldots\right] \varepsilon^{1 / 2}\right] \mathbf{z}_{n}(\alpha, \omega) \\
& =\Omega_{n}^{2}(\alpha, \omega) \mathbf{z}_{n}(\alpha, \omega) .
\end{aligned}
$$

Eq. (99) is a an eigenvalue equation that is nonlinear in $\omega$. If the frequencies $\omega$ equal the square root $\Omega_{n}$ of an eigenvalue then this $\Omega_{n}$ is also an eigenvalue of Eq. (97) that can be interpreted as excitation energy. In this sense Eq. (99) is equivalent to Eq. (97) the basis of the considered hybrid method. Eq. (99) has exactly the form the basic equation (35) of TDDFT. The contributions $[\alpha(\boldsymbol{\Delta}+\mathbf{A}+$ B) $-\alpha \omega^{2}(\boldsymbol{\Delta}+\mathbf{A}-\mathbf{B})$ that are linear in $\alpha$ represent the Hartree and the exact frequency-dependent exchange kernel. The terms of higher order in $\alpha$ have to be interpreted as frequency-dependent contributions of the correlation kernel because only the correlation kernel contains contributions of quadratic and higher order in $\alpha$. Because terms up to infinite order in $\alpha$ are contained in Eq. (99) and thus taken into account in the EXX- and AC-RPA hybrid method based on Eq. (99) we call this method EXX-RPA[ $\infty]$ here.

The correlation energy both of the EXX-RPA and the EXX-RPA $[\infty]$ method can be written in the general form

$$
E_{c}^{\mathrm{EXX}-\mathrm{RPA}}=\frac{1}{2} \int_{0}^{1} d \alpha \operatorname{Tr}\left[\mathbf{C}\left(\mathbf{U}_{\alpha} \mathbf{U}_{\alpha}^{T}-\mathbf{1}\right)\right]=\frac{1}{2} \int_{0}^{1} d \alpha \operatorname{Tr}[\mathbf{C P} \alpha]
$$

with $\mathbf{U}_{\alpha}$ containing in its columns the eigenvectors $\mathbf{u}_{n}^{\alpha}=\left(\omega_{n}^{\alpha}\right)^{-1 / 2} \varepsilon^{1 / 2} \mathbf{z}_{n}^{\alpha}$ for a given coupling strength $\alpha$, compare Eqns. (76) and (39) where the eigenvectors $\mathbf{z}_{n}^{\alpha}$ in case of EXX-RPA are obtained from Eq. (36) and in case of EXX-RPA[ $\infty]$ from 
Eq. (97) or (99), respectively. It has been shown in Ref. [53] that the EXX-RPA correlation energy according to Eq. (100) reproduces the correct second-order energy of perturbation theory along the adiabatic connection [116, 117] apart from the missing singles term due to the assumption that the EXX and Hartree-Fock orbitals are obtained by an occupied-occupied virtual-virtual unitary transformation only. While this can be shown to hold true also for the EXX-RPA[ $\infty]$ method, the corresponding third-order correlation energies differ in both cases. More precisely, it can be shown that the third-order expansion of the EXX-RPA correlation energy can not be cast into the particle-hole terms of the form of those given in Eq. (93) or table 1. On the other hand, for the EXX-RPA[ $\infty]$ approach one obtains the same third-order correlation energy terms as for the AC-RPA method (written in terms of EXX orbitals and eigenvalues, however), accompanied by the four additional contributions $\left(2 b^{\mathrm{lx}}\right),\left(2 \mathrm{~b}^{\mathrm{lx}}\right),\left(2 \mathrm{~g}^{\mathrm{lx}}\right)$ and $\left(2 \mathrm{~h}^{\mathrm{lx}}\right)$ steming from $\boldsymbol{\Delta}$ matrix elements, see table 1 . As will be demonstrated in section 5 , the additional terms in EXX-RPA and EXX-RPA $[\infty]$ approaches will yield significant contributions to the total correlation energy. Thus exchange RPA methods using a Kohn-Sham reference determinant should account for these non-standard contributions in practical calculations. Note, however, that this is not true for the SOSEX variant, since, by definition, here no exchange contributions are accounted for in the calculation of the response function, see section 3.2.

It has been shown [55] that the EXX-RPA correlation energies are distinctively different from those from the AC-RPA method. The reason for this is that, while the coupling-strength integrand $\mathbf{C P}_{\alpha}$ in both cases is similar for $\alpha \rightarrow 0$ (where $\mathbf{C P}_{\alpha} \rightarrow \mathbf{0}$ ) and $\alpha \rightarrow 1$, for coupling strengths between 0 and 1 the partitioning into an interacting and noninteracting response function leads to significant differences in both cases, especially in cases where the static correlation is dominating [55].

The analogue to the NRPA1 (rCCD) method using Kohn-Sham exchange will yield the same expression for the correlation energy

$$
E_{c}^{\mathrm{EXX}-\mathrm{NRPA} 1}=\frac{1}{4} \operatorname{Tr}\left(\mathbf{B Y X}^{-1}\right)
$$

but the eigenvector components $\mathbf{X}$ and $\mathbf{Y}$ are obtained from the supermatrix form of the TDHF eigenvalue equation written in terms of EXX orbitals and eigenvalues:

$$
\left(\begin{array}{cc}
\varepsilon+\Delta+\mathbf{A} & \mathbf{B} \\
-\mathbf{B} & -\varepsilon-\Delta-\mathbf{A}
\end{array}\right)\left(\begin{array}{l}
\mathbf{X} \\
\mathbf{Y}
\end{array}\right)=\omega\left(\begin{array}{l}
\mathbf{X} \\
\mathbf{Y}
\end{array}\right)
$$

It has recently been shown by Jansen et al. [114] that the NRPA1 correlation energy is identical to following expression (see also section 3.2):

$$
E_{c}^{\mathrm{NRPA} 1}=\frac{1}{4} \int_{0}^{1} d \alpha \operatorname{Tr}\left[\mathbf{B} \mathbf{P}_{\alpha}\right]+\operatorname{Tr}\left[(\mathbf{A}-\mathbf{B})\left(\mathbf{X}_{\alpha} \mathbf{X}_{\alpha}^{T}+\mathbf{Y}_{\alpha} \mathbf{Y}_{\alpha}^{T}-\mathbf{1}\right]\right.
$$

Since in the local exchange case the relation

$$
\Delta+\mathbf{A}-\mathbf{B} \approx \mathbf{0}
$$

holds true, which implies the closeness between the adiabatic and non-adiabatic 
form of the eigenvalue Eq. (99), the EXX-RPA1 correlation energy from Eq. (101) then can also be approximated as

$$
E_{c}^{\mathrm{EXX}-\mathrm{NRPA} 1} \approx \frac{1}{4} \int_{0}^{1} d \alpha \operatorname{Tr}\left[\mathbf{B P}_{\alpha}\right]
$$

which directly links the NRPA1 (rCCD) correlation energy to an adiabatic connection formula that differs, however, from Eq. (100) due to the occurrence of antisymmetrised integrals in Eq. (105). Performing the same transformation as between Eq. (46) and Eq. (74) one arrives at the expression

$$
E_{c}^{\mathrm{EXX}-\mathrm{NRPA} 3} \approx \frac{1}{2} \int_{0}^{1} d \alpha \operatorname{Tr}\left[\mathbf{C P}_{\alpha}\right]
$$

which is identical to the EXX-RPA correlation energy expression in Eq. (100) and which is termed EXX-NRPA3 as it is found that it gives practically the same correlation energy as the NRPA3 approach (Eq. (69)) in terms of local Kohn-Sham exchange

$$
E_{c}^{\mathrm{EXX}-\mathrm{NRPA} 3}=\frac{1}{2} \operatorname{Tr}\left(\left[{ }^{1} \mathbf{B}-{ }^{3} \mathbf{B}\right]{ }^{1} \mathbf{Y}^{1} \mathbf{X}^{-1}\right)=\frac{1}{2} \operatorname{Tr}\left(\mathbf{C}^{1} \mathbf{Y}^{1} \mathbf{X}^{-1}\right)
$$

A reason for the similarity between Eqs. (100) and (107) can be deduced using the perturbation theory analysis from the last section. Considering the second-order response propagator of Eq. (91) and using $\boldsymbol{\Delta}+\mathbf{A} \approx \mathbf{B}$ (note that $\boldsymbol{\Delta}+\mathbf{A}=\mathbf{B}$ for two-electron systems) which follows from Eq. (104), then all terms in Eq. (91) are approximately identical (substitute $\mathbf{A}$ by $\boldsymbol{\Delta}+\mathbf{A}$ in the equation). In case of the adiabatic connection method the number of terms contributing to $\mathbf{P}^{(2)}$ is 6 (since two of the diagonal contributions are zero after frequency integration) while in case of EXX-NRPA3 one only accounts for the nondiagonal terms in Eq. (91), that is 4 . Since in EXX-RPA the prefactor in third order is $\frac{1}{3}$ and in case of EXX-NRPA3 it is $\frac{1}{2}$ both methods have the same number of terms in that order and thus it holds true that the third-order contribution to the response propagator is approximately identical for EXX-RPA and EXX-NRPA3 (in case of two-electron systems it is exactly equal to each other).

Since the EXX-NRAP3 correlation energy of Eq. (106) should be identical to the NRPA3 correlation energy of Eq. (69) if the Hartree-Fock and EXX determinants can be transformed into each other through occupied-occupied unitary transformations, we can derive the following energy relationship:

$$
E_{c}^{\mathrm{NRPA} 3} \approx E_{c}^{\mathrm{EXX}-\mathrm{NRPA} 3} \approx E_{c}^{\mathrm{EXX}-\mathrm{RPA}}
$$

Correspondingly, the AC-RPA correlation energy of Eq. (76) expressed in terms of the solutions of the TDHF Eq. (94) and the AC-RPA correlation energy of Eq. (76) evaluated with the solution vectors of Eq. (96), a TDHF equation expressed in terms of EXX orbitals, are similar to each other due to the similarity of the EXX and Hartree-Fock determinants. The latter approximation, i.e., the evaluation of the AC-RPA correlation energy expression (76) with vectors $\mathbf{x}_{n}+\mathbf{y}_{n}=\mathbf{z}_{n}$ and excitation energies $\Omega_{n}$ obtained from the TDHF equation expressed in EXX orbitals, Eq. (96), shall be denoted AC-RPA(EXX). Differences between AC-RPA and AC- 
RPA(EXX) correlation energies are caused exclusively by the small differences of EXX and HF determinants. This will be further discussed considering numerical results in the sections 5 and 6 .

\section{Total energies from RPA correlation functionals}

Total energies have been calculated for a range of small molecules shown in figure 1 using the aug-cc-pVTZ basis set of Dunning et al. [118]. The geometries for the molecules were optimised at the MP2 level using the aug-cc-pVTZ basis set and are taken from Ref. [119]. The exact-exchange Kohn-Sham (EXX) calculations were done in two steps: firstly the local exchange potential was calculated using the uncontracted triple-zeta auxiliary and orbital basis sets from Ref. [75]. In the second step these exchange potentials were used in a subsequent Kohn-Sham calculation with the smaller contracted aug-cc-pVTZ basis set in which only the Coulomb potential was optimised self-consistently. Direct RPA calculations were also performed using orbitals and eigenvalues from the Perdew-Burke-Ernzerhof (PBE) exchange-correlation functional [120]. Note that in this case the exchange energy was calculated using the exact exchange energy functional in terms of PBE orbitals. Core electrons were correlated in the calculations. All calculations were done using the developers version of the Molpro quantum chemistry program [121].

A comparsion of total energies for RPA methods based on Hartree-Fock with total energies from other correlation methods can be found elsewhere [112]. Here we will focus mainly on the RPA methods based on Kohn-Sham reference determinants. Figure 1 displays the energy differences of several RPA methods to CCSD $(\mathrm{T})$ reference values. Note that the methods EXX-RPA and EXX-RPA[ $\infty]$ are excluded in the diagram, since they yield energy differences that are indistinguishable from the EXX-NRPA3 values on the scale of figure 1, see below. It can be seen that with exception of EXX-NRPA3 and EXX-SOSEX all other methods overestimate the $\operatorname{CCSD}(\mathrm{T})$ correlation energy. (Note that the correlation energy for Kohn-Sham based RPA approaches includes also a small contribution due to the differences of the EXX/PBE and Hartree-Fock determinants.) For PBE-dRPA and EXX-dRPA one can observe the strongest deviations to the $\operatorname{CCSD}(\mathrm{T})$ values, which is due to the large self-correlation errors in both methods. One can see that the EXX-dRPA and PBE-dRPA results are very similar to each other and thus it can be stated that the dRPA funtional is relatively insensitive with respect to the choice of the KS reference determinant. Note that in case of PBE-dRPA the exchange energy is calculated in the same way as in EXX based methods, that is, by evaluating the exact exchange energy expression with PBE orbitals. While the dRPA self-correlation error is corrected to some extend by all other RPA methods including exchange effects (beyond first order) displayed in figure 1, the EXX-NRPA1, EXX-NRPA2 and EXX-NRPA4 methods still strongly underestimate the CCSD(T) energies,i.e., strongly overestimate the magnitude of the correlation energy. An analysis of the third-order contribution of the correlation energy shows that this is due to the strong negative third-oder correlation terms $(2 \mathrm{~b})$ and $(2 \mathrm{~h})$ that more than quench the positive direct term (2a), see tables 2 and 1 (see also Ref. [112]). In case of EXX-SOSEX these third-order terms are absent, see table 2, but the only thirdorder exchange term (2e) in the SOSEX method is generally much smaller than the (2a) term, so that the CCSD(T) energies are underestimated by EXX-SOSEX for the molecules shown in figure 1 . The best agreement of the considered RPA methods with the CCSD(T) energies is obtained with the EXX-NRPA3 method and thus also with the EXX-RPA and EXX-RPA $[\infty]$ methods that yield almost identical results, as already mentioned above. The third-order analysis from table 
2 shows that the NRPA3 method contains the (2b) term but misses the (2h) contribution and therefore its third-order correlation contribution is generally much smaller than with the EXX-NRPA1, EXX-NRPA2 and EXX-NRPA4 methods.

In figure 2 the differences of the total energies of the methods EXX-NRPA3, EXX-RPA, EXX-RPA[ $\infty]$, AC-RPA, and AC-RPA(EXX) to the NRPA3 energies are shown for the range of molecules. As already anticipated in section 4, the total energies of the EXX-NRPA3, EXX-RPA, EXX-RPA[ $[$ ], and NRPA3 methods do not differ by more than 1-4 millihartree on average. Interestingly the diagram in figure 2 shows, that the EXX-RPA[ $\infty]$ energies are even closer to the NRPA3 energies than the EXX-NRPA3 energies although the methods are not directly related to each other by unitary orbital transformations. The largest deviations to the NRPA3 energies is found for EXX-RPA. Figure 2 also contains the energy differences for the AC-RPA and AC-RPA(EXX) methods to NRPA3. Going from the AC-RPA over the AC-RPA(EXX) and the EXX-RPA[ $\infty]$ to the EXX-RPA method the importance of various changes between the methods can be considered step by step. Differences between the AC-RPA and the AC-RPA(EXX) method are small and due to the only small differences between EXX and HF determinants,

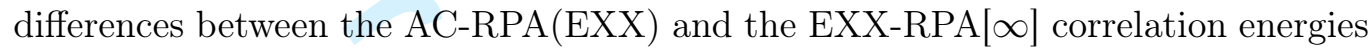
are more substantial and are due to the fact whether or not the matrix $\Delta$ in Eq. (96) and Eq. (97) are scaled with the coupling constant or not. This scaling obviously

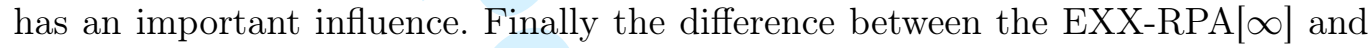
the EXX-RPA correlation energies is small again. This difference is due to the difference between the right hand sides of Eq. (97) and the TDEXX equation (36) which are only of second order in $\varepsilon^{-1 / 2}[\mathbf{A}-\mathbf{B}+\boldsymbol{\Delta}] \varepsilon^{-1 / 2}$. In the next section it will be investigated whether these energy differences affect energy differences for a set of chemical reactions.

Figure 3 displays the correlation energies for Hartree-Fock based and EXX-based RPA methods for the molecules $\mathrm{CH}_{4}$ (top) and $\mathrm{HCOOH}$ (bottom) together with the corresponding sum of the second- and third-order contributions in each case. It can be observed that the NRPA1, NRPA3 and NRPA4 correlation energies are very close to the corresponding EXX-NRPA1, EXX-NRPA3 and EXX-NRPA4 correlation energies. This, again, can be explained by the closeness of the RPA amplitudes due to the similarity between the time-dependent Hartree-Fock and time-dependent EXX equations. In contrast to this, the NRPA2 and EXX-NRPA2 correlation energies differ strongly from each other, because the second-order correlation energy contained in the energy expression for EXX-NRPA2 (see Eq. (66)) differs strongly if evaluated with EXX or Hartree-Fock orbitals due to the different orbital energies in both cases. The differences of the orbital eigenvalue spectrum are also the reason why one can observe huge differences of the correlation energies for the dRPA and SOSEX methods in the diagrams in figure 3. Since the single particle transition energies in EXX are generally lower than in Hartree-Fock theory due to the self-interaction correction for the virtual states, the EXX-dRPA and EXX-SOSEX correlation energies are considerably larger in magnitude than the corresponding dRPA and SOSEX correlation energies, respectively. In case of adiabatic connection RPA methods a significant difference between the AC-RPA and EXX-RPA[ $\infty]$ can be seen in figure 3 which is due to the differences between the TDHF and TDEXX response functions at coupling strengths between 0 and 1, compare with Ref. [55] where the dsifferences between AC-RPA and EXX-RPA is discussed. A comparison of the total correlation energies with the corresponding sum of the secondand third-order correlation terms shows that in case of the Hartree-Fock based RPA methods the higher order correlation contributions, given by the difference $E_{\mathrm{c}}-E_{\mathrm{c}}^{(2)}-E_{\mathrm{c}}^{(3)}$, are, with exception of the NRPA2 values, rather small, while in 
case of the EXX based methods larger higher order correlation contributions can be observed. Thus the perturbation expansion of the RPA correlation energy is not that well converged in low orders if calculated with KS orbitals.

\section{Reaction energies from RPA correlation functionals}

The total energies for the set of molecules shown in figure 1 were used to calculate the reaction energies for a set of 16 chemical reactions listed in the first column in table 3.

Table 3 shows a comparison between the NRPA3, EXX-NRPA3, EXX-RPA and EXX-RPA $[\infty]$ methods as well as for the AC-RPA and AC-RPA(EXX) methods for the set of chemical reactions. The last colum in table 3 contains the $\operatorname{CCSD}(\mathrm{T})$ reference data from Ref. [53]. It can be seen that in spite of slight deviations in the total energies between the different RPA methods, see table 2, their reaction energies for the given chemical reactions are basically identical among the NRPA3, EXX-NRPA3, EXX-RPA and EXX-RPA[ $\infty]$ methods on the one hand and among AC-RPA and AC-RPA(EXX) on the other hand. The root-mean squared (rms) errors and mean-absolute (mae) errors to the $\operatorname{CCSD}(\mathrm{T})$ reaction energies all differ by no more than 0.3 millihartree, see last two lines in table 3 . Therefore one can conclude that one can practically expect the same accuracy from the NRPA3, EXXNRPA3, EXX-RPA and EXX-RPA[ $\infty]$ methods and correspondingly for AC-RPA and AC-RPA(EXX) in quantum chemistry applications.

In figure 4 the rms errors (top diagram) and relative percentual deviations (bottom diagram) of various Kohn-Sham orbital based RPA methods to the CCSD(T) reference reaction energies are shown. Along with the errors for the RPA methods, the diagrams in figure 4 also contain the corresponding error bars for Hartree-Fock, MP2 (second-order Møller-Plesset) and CCSD for comparison. Note that the errors for some RPA methods (EXX-NRPA1, EXX-NRPA2 and EXX-NRPA4) are not shown due to their strong differences to the $\operatorname{CCSD}(\mathrm{T})$ values which are even worse than Hartree-Fock errors. For the RPA methods shown in figure 4 one can observe rms errors that are consistently smaller than with MP2, but all methods have larger average errors than CCSD. Interestingly, the PBE-dRPA, EXX-dRPA and EXX-SOSEX methods yield, for the chemical reactions considered, about the same accuracy as the EXX-RPA method in spite of strongly differing total energies, see section 5: for EXX-dRPA the rms error is only about $0.2 \mathrm{kcal} / \mathrm{mol}$ larger than with EXX-RPA and the percentual deviation, which more emphasises reactions with small reaction energies, is even about 1\% smaller compared to EXX-RPA. The SOSEX approach, which corrects the dRPA correlation method in second (and higher) order, does not lead to an improvement of the dRPA values for the set of chemical reactions.

\section{Summary}

Starting from a relation between the two-particle density matrix and the response function of the many-body system, expressions for the correlation energy of electronic systems have been given in terms of the interacting and noninteracting response functions. This relation, called fluctuation-dissipation theorem, represents a general way to obtain correlated ground-state properties of an interacting manybody system and solely depends on approximations of the response function. By combining this ansatz with a coupling-constant integration along the adiabatic connection the adiabatic-connection fluctuation-dissipation theorem is obtained which 
provides an exact expression for the correlation energy as defined in the Kohn-Sham formalism of density-functional theory.

A first approximation to the interacting response function is obtained by taking Coulomb interactions between the particles into account. This approximation is termed as direct random-phase approximation (dRPA) and has most often been used for the description of solid states in the past. It turns out that, at least if a Hartree-Fock (HF) reference state is used, total dRPA correlation energies are not very accurate due to a large self-correlation error. An advantage of RPA based on a Kohn-Sham (KS) reference determinant is that errors may be somewhat reduced since a KS description of the reference state can mimic the effect of missing singly excited determinants in RPA methods.

In order to correct the self-correlation error of dRPA, exchange interactions have to be accounted for in second and higher orders, that is, the electron interaction operator has to be complemented by an additional exchange kernel. It has been shown that several RPA methods can be derived that include exchange interactions. While all of them yield, in a perturbation series expansion, the correct second-order expression known from many-body perturbation theory, they differ in third-order of perturbation theory. An analysis of the different RPA methods based both, on HF and exact Kohn-Sham exchange (EXX) determinants reveals that the differences of the correlation energies in third order lead also to strong differences in the total correlation energies of these methods. A comparison with coupled-cluster singles doubles with perturbative triples $(\mathrm{CCSD}(\mathrm{T}))$ energies shows, that with exception of the (in this work termed) NRPA3 and adiabatic connection RPA method the other RPA variants considered in this work yield large errors for the correlation energies for a set of small molecules. It has been shown that for RPA based on the EXX determinant, the corresponding EXX-NRPA3 and EXX-RPA (the adiabatic connection RPA method in an EXX KS formalism) are closely related to each other and yield similar total energies. Since the (HF based) NRPA3 and the EXXNRPA3 method too give similar results due to the closeness between the HF and EXX determinants, also a relation between the HF based NRPA3 method and the EXX based EXX-RPA method can be derived.

The accuracy of the different RPA methods considered in this work has also been tested for the description of reaction energies for a set of 16 chemical reactions for some small organic molecules. By comparing the results with accurate CCSD(T) reference values, it was found that the methods that have the smallest errors for the total energies, namely NRPA3 and EXX-RPA, also give the best agreement for reaction energies. The, historically, older RPA methods termed as NRPA1 (identical to the ring coupled-cluster doubles method), NRPA2 and NRPA4 here, yield large errors also for the description of reaction energies. In contrast to this, the KS orbital based dRPA and SOSEX approach produce reaction energies that are not much worse than reaction energies energies from NRPA3 or EXX-RPA methods and even slightly better than with second-order Møller-Plesset perturbation theory. This result may be interesting from a practical point of view, since dRPA and SOSEX methods can be implemented in a more efficient way than NRPA3 and EXX-RPA methods.

Generally it can be concluded that the use of Kohn-Sham orbitals in RPA methods instead of Hartree-Fock orbitals offers new possibilities to make RPA methods both, more accurate and also more efficient for the description of correlated molecular ground states. The first point may be true, since the Kohn-Sham orbitals can be expected to be closer to variationally optimised orbitals within RPA methods, since it has been shown that, depending on the underlying exchange-correlation potentials, KS orbitals are closer to Brueckner orbitals from a coupled-cluster doubles 
wave function than HF ones [88]. The second argument regarding the efficiency to date holds true especially for solids for which direct RPA methods have been implemented with a low computational cost [40]. In case of RPA methods for molecules the development of efficient computer programs is a field of active research and a number of different methods were already presented in recent years [39, 45].

\section{Acknowledgments}

We gratefully acknowledge the funding of the German Research Council (DFG), which, within the framework of its 'Excellence Initiative', supports the Cluster of Excellence 'Engineering of Advanced Materials' (www.eam.uni-erlangen.de) at the University of Erlangen-Nuremberg. We are grateful to Wim Klopper for providing us with Ref. [31] prior to its publication.

\section{References}

1]D. Bohm and D. Pines, Phys. Rev. 82, 625 (1951).

[2]D. Pines and D. Bohm, Phys. Rev. 85, 338 (1952).

[3] D. Bohm and D. Pines, Phys. Rev. 92, 609 (1953).

[4]P. Nozieres and D. Pines, Nuovo Cim. 9, 470 (1958).

[5]H. Ehrenreich and M.H. Cohen, Phys. Rev. 115, 786 (1959).

[6] K. Sawada, K.A. Brueckner and N. Fukuda, Phys. Rev. 108, 507 (1957).

[7]F. London, Z. Physik. Chem. B 11, 222 (1930).

[8]F. London, Trans. Faraday Soc. 33, 8 (1937).

[9] M. Gell-Mann and K.A. Brueckner, Phys. Rev. 106, 364 (1957).

[10] N.E. Brener and J.L. Fry, Phys. Rev. B 19, 1720 (1979).

[11]N.E. Brener and J.L. Fry, Phys. Rev. B 22, 2737 (1980).

[12] J. Lindhard, K. Dan. Vidensk. Selsk. Mat. Fys. Medd. 28, 8 (1954).

[13] A.D. MacLachlan and M.A. Ball, Rev. Mod. Phys. 36, 844 (1964).

14] M.A. Ball and A.D. McLachlan, Mol. Phys. 7, 501 (1964).

[15] G. Csanak, J. Phys. B: Atom. Molec. Phys. 7, 1289 (1974).

[16]P. Jørgensen, J. Oddershede and M. Ratner, J. Chem. Phys. 61, 710 (1974).

[17] G.P. Arrighini and C. Guidotti, J. Phys. B: Atom. Molec. Phys. 7, 1790 (1974).

[18]P. Jørgensen, Annu. Rev. Phys. Chem. 26, 359 (1975).

[19] J. Oddershede, P. Jørgensen and D.L. Yeager, Comp. Phys. Rep. 2, 33 (1984).

[20]H. Weiss, R. Ahlrichs and M. Häser, J. Chem. Phys. 99, 1262 (1993).

[21]T.H. Dunning and V. McKoy, J. Chem. Phys. 47, 1735 (1967).

[22] J. Cizek and J. Paldus, J. Chem. Phys. 47, 3976 (1967).

[23] K.D. Jordan, Int. J. Quant. Chem. 7, 491 (1973).

[24] J. Oddershede, P. Jørgensen and N.H.F. Beebe, J. Chem. Phys. 63, 2996 (1975).

[25] G. Chambaud, B. Levy and P. Millie, Theoret. Chim. Acta 48, 103 (1978).

[26] D. Pines and P. Nozieres, The theory of quantum liquids, Vol. I: Normal Fermi liquids W. A. Benjamin, NY, Amsterdam.

[27]J. Harris and A. Griffin, Phys. Rev. B 11, 3669 (1975).

[28]D.C. Langreth and J.P. Perdew, Solid State Commun. 17, 1425 (1975).

[29] D.C. Langreth and J.P. Perdew, Phys. Rev. B 15, 2884 (1977).

[30] H.B. Callen and T.A. Welton, Phys. Rev. 83, 34 (1951).

[31]W. Klopper, A.M. Teale, S. Coriani, T.B. Pedersen and T. Helgaker, Chem. Phys. Lett. (2011), submitted.

[32]T. Kotani, J. Phys.: Condens. Matter 10, 9241 (1998).

[33]F. Furche, Phys. Rev. A 64, 195120 (2001).

[34] T. Miyake, F. Aryastiawan, T. Kotani, M. van Shilfgaarde, M. Usuda and K. Terakura, Phys. Rev. B 66, 245103 (2002).

[35] M. Fuchs and X. Gonze, Phys. Rev. B 65, 235109 (2002).

[36]Y.M. Niquet and X. Gonze, Phys. Rev. B 70, 245115 (2004).

[37] M. Fuchs, Y.M. Niquet, X. Gonze and K. Burke, J. Chem. Phys. 122, 094116 (2005).

[38] H. Jiang and E. Engel, J. Chem. Phys. 127, 184108 (2007).

[39]F. Furche, J. Chem. Phys. 129, 114105 (2008).

[40]H.V. Nguyen and S. de Gironcoli, Phys. Rev. B 79, 205114 (2009).

[41]A. Grüneis, M. Marsman, J. Harl, L. Schimka and G. Kresse, J. Chem. Phys. 131, 154115 (2009).

[42] J. Toulouse, I. C. Gerber, G. Jansen, A. Savin and J. G. Angyan, Phys. Rev. Lett. 102, 096404 (2009).

[43]B.G. Janesko, T.M. Henderson and G.E. Scuseria, J. Phys. Chem. 130, 081105 (2009).

[44] M. Hellgreen and U. von Barth, J. Chem. Phys. 131, 044110 (2009).

[45]H. Eshuis, J. Yarkony and F. Furche, J. Chem. Phys. 132, 234114 (2010).

[46]J. Harl, L. Schimka and G. Kresse, Phys. Rev. B 81, 115126 (2010).

[47]H.V. Nguyen and G. Galli, J. Chem. Phys. 132, 044109 (2010). 
[48]D. Lu, H.V. Nguyen and G. Galli, J. Chem. Phys. 133, 154110 (2010).

[49] W. Zhu, J. Toulouse, A. Savin and J. G. Angyan, J. Chem. Phys. 132, 244108 (2010).

[50] J. Toulouse, W. Zhu, J. G. Angyan and A. Savin, Phys. Rev. A 82, 032502 (2010).

[51] J. Paier, B.G. Janesko, T.M. Henderson, G.E. Scuseria, A. Grüneis and G. Kresse, J. Chem. Phys. 132, 094103 (2010), Erratum: J. Chem. Phys. 133 (2010) 179902.

[52] M. Hellgreen and U. von Barth, J. Chem. Phys. 132, 044101 (2010).

[53] A. Heßelmann and A. Görling, Mol. Phys. 108, 359 (2010).

[54]X. Ren, A. Tkatchenko, P. Rinke and M. Scheffler, arXiv:1011.2724v1 [cond-mat.other] (2010).

[55]A. Heßelmann and A. Görling, Phys. Rev. Lett. 106, 093001 (2011).

[56]F.E. Harris, H.J. Monkhorst and D.L. Freeman, Algebraic and diagrammatic methods in manyfermion theory $(,, 1992)$.

[57] J. Harl and G. Kresse, Phys. Rev. Lett. 103, 056401 (2009).

[58]T. Leininger, H. Stoll, H.J. Werner and A. Savin, Chem. Phys. Lett. 275, 151 (1997).

[59] J.G. Ángyán, I.C. Gerber, A. Savin and J. Toulouse, Phys. Rev. A 72, 012510 (2005).

[60] J. Toulouse, F. Colonna and A. Savin, Phys. Rev. A 70, 062505 (2004).

[61]E. Goll, H.J. Werner, H. Stoll, T. Leininger, P. Gori-Giorgi and A. Savin, Chem. Phys. 329, 276 (2006).

[62]B.G. Janesko, T.M. Henderson and G.E. Scuseria, J. Phys. Chem. 131, 034110 (2009), Erratum: J. Phys. Chem. 133 (2010) 179901.

[63]B.G. Janesko and G.E. Scuseria, J. Chem. Phys. 131, 154106 (2009).

[64]F. Furche and T. Van Voorhis, J. Chem. Phys. 122, 164106 (2005).

[65]M. Hellgren and U. von Barth, Phys. Rev. B 78, 115107 (2008).

[66] A. Görling, Int. J. Quantum Chem. 69, 265 (1998).

[67] A. Görling, Phys. Rev. A 57, 3433 (1998).

[68] Y.H. Kim and A. Görling, Phys. Rev. B 66, 035144 (2002).

69] A. Heßelmann, A. Ipatov and A. Görling, Phys. Rev. A 80, 012507 (2009).

[70] A. Ipatov, A. Heßelmann and A. Görling, Int. J. Quantum Chem. 110, 2202 (2010).

[71] A. Heßelmann and A. Görling, J. Chem. Phys. 134, 034120 (2011).

[72] J. Harris and R.O. Jones, J. Phys. F 4, 1170 (1974).

[73] O. Gunnarson and B.I. Lundquvist, Phys. Rev. B 13, 4274 (1976).

[74]F. Della Sala and A. Görling, J. Chem. Phys. 115, 5718 (2001).

[75] A. Heßelmann, A.W. Götz, F. Della Sala and A. Görling, J. Chem. Phys. 127, 054102 (2007).

[76] A. Görling, A. Ipatov, A.W. Götz and A. Heßelmann, Z. Phys. Chem. 224, 325 (2010).

[77]M. Lein, J.F. Dobson and E.K.U. Gross, J. Comp. Chem. 20, 12 (1999).

[78] M.E. Casida, in (, , 1995), Vol. I, p. 155

[79]E.K.U. Gross, J.F. Dobson and M. Petersilka, in (, , 1996), Vol. 181, p. 81.

[80] A. Görling, H. Heinze, S.P. Ruzankin, M. Staufer and N. Rösch, J. Chem. Phys. 110, 2785 (1999).

[81]F. Furche, J. Chem. Phys. 114, 5982 (2001).

[82] M. A. L. Marques et al., editor, Time-Dependent Density Functional Theory, Lecture Notes in Physics, Vol. $706(,, 2006)$

[83]P. Elliot, F. Furche and K. Burke, Rev. Comp. Chem. 26, 91 (2009).

[84] J. Oddershede, Adv. Quant. Chem. 11, 275 (1978).

[85]R.M. Dreizler and E.K.U. Gross, Density Functional Theory (, , 1990).

[86] L.Z. Stolarczyk and H.J. Monkhorst, Int. J. Quant. Chem. Symp. 18, 267 (1984).

[87] A. Heßelmann and G. Jansen, Chem. Phys. Lett. 315, 248 (1999).

[88] A. Heßelmann, Ph.D. thesis, Universität Duisburg-Essen 2003.

[89]R.D. Mattuck, A guide to Feynman diagrams in the many-body problem (, , 1992).

[90]R. Moszynski, B. Jeziorski and K. Szalewicz, Int. J. Quant. Chem. 45, 409 (1993).

[91]N. Fukuda, F. Iwamoto and K. Sawada, Phys. Rev. 135, A932 (1964).

[92]N. Ostlund and M. Karplus, Chem. Phys. Lett. 11, 450 (1971).

[93] A. Szabo and N.S. Ostlund, J. Chem. Phys. 67, 4351 (1977).

[94] G.E. Scuseria, T.M. Henderson and D.C. Sorensen, J. Chem. Phys. 129, 231101 (2008).

[95]D.J. Rowe, Rev. Mod. Phys. 40, 153 (1968).

[96]R. McWeeny, Methods of molecular quantum mechanics (, , 1996).

[97]D.B. Cook, Handbook of Computational Quantum Chemistry (, , 2004).

[98]D. Pines, The many-body problem (, , 1962).

[99] J. Linderberg, Int. J. Quant. Chem. Symp. S1, 719 (1967).

[100] J. Oddershede and P. Jørgensen, J. Chem. Phys. 66, 1541 (1977).

[101]E.A. Sanderson, Phys. Lett. 19, 141 (1965).

[102]T.I. Shibuya and V. McKoy, J. Chem. Phys. 53, 3308 (1970).

[103] T.I. Shibuya and V. McKoy, Phys. Rev. A 2, 2208 (1970).

[104] T.I. Shibuya and V. McKoy, J. Chem. Phys. 54, 1738 (1971).

[105] J. Linderberg, P. Jørgensen, J. Oddershede and M. Ratner, J. Chem. Phys. 56, 6213 (1972).

[106] A.C. Lasaga and M. Karplus, Phys. Rev. A 16, 807 (1977).

[107]A.C. Lasaga and M. Karplus, J. Chem. Phys. 71, 1218 (1979).

[108] M.J. Packer, E.K. Dalskov, T. Enevoldsen, H.J.A. Jensen and J. Oddershede, J. Chem. Phys. 105, $5886(1996)$

[109]J. Oddershede, N.E. Grüner and G.H.F. Diercksen, Chem. Phys. Lett. 97, 303 (1985).

[110] T. Shibuya, J. Rose and V. McKoy, J. Chem. Phys. 58, 500 (1973).

[111]P. Jørgensen, J. Oddershede and M. Ratner, Chem. Phys. Lett. 32, 111 (1975).

[112] A. Heßelmann, J. Chem. Phys. 134, 204107 (2011).

[113] A. Szabo and N.S. Ostlund, Int. J. Quant. Chem. S11, 389 (1977).

[114] G. Jansen, R.F. Liu and J. G. Angyan, J. Chem. Phys. 133, 154106 (2010).

[115] A. Heßelmann and A. Görling, Chem. Phys. Lett. 455, 110 (2008).

[116]A. Görling and M. Levy, Phys. Rev. B 47, 13105 (1993). 


\section{Molecular Physics}

[117]A. Görling and M. Levy, Int. J. Quant. Chem. Symp. 29, 93 (1995).

[118] T.H. Dunning, J. Chem. Phys. 90, 1007 (1989).

[119] H.J. Werner, T.B. Adler and F.R. Manby, J. Chem. Phys. 126, 164102 (2007).

[120] J.P. Perdew, K. Burke and M. Ernzerhof, Phys. Rev. Lett. 77, 3865 (1996).

[121]H.J. Werner, P.J. Knowles, R. Lindh, F.R. Manby, M. Schütz et al., MOLPRO, version 2010.2, a package of ab initio programs Cardiff, UK, 2010, see http://www.molpro.net. 
Table 1. Second- and third-order correlation energy terms. The definition $\bar{M}_{i a, j b}=\frac{M_{i a, j b}}{\varepsilon_{i a}+\varepsilon_{j b}}$ for a matrix M has been used. The matrix elements are defined as $C_{i a, j b}=\langle i j \mid a b\rangle, J_{i a, j b}=\langle i a \mid j b\rangle, K_{i a, j b}=\hat{P}_{a b} C_{i a, j b}$ (the permutation operator $\hat{P}_{a b}$ exchanges indices $a$ and $\left.b\right), \Delta_{i a, j b}=\delta_{a b}\left\langle i\left|v_{\mathrm{x}}^{\mathrm{NL}}-v_{\mathrm{x}}\right| j\right\rangle-\delta_{i j}\left\langle a\left|v_{\mathrm{x}}^{\mathrm{NL}}-v_{\mathrm{x}}\right| b\right\rangle\left(v_{\mathrm{x}}^{\mathrm{NL}}\right.$ : nonlocal exchange potential, $v_{\mathrm{x}}$ : local exchange potential) and $\varepsilon_{i a}=\varepsilon_{a}-\varepsilon_{i}$. In the terms (2i)-(2l) the matrix elements are defined according to $E_{a b}^{c d}=\langle a d \mid c b\rangle$ and $S_{i j}^{k l}=\langle i j \mid k l\rangle$. The terms (1a) and (1b) correspond to the direct and exchange part of the second-order correlation energy, terms $(2 \mathrm{a})-(2 \mathrm{~h})$ are particle-hole terms and terms (2i)-(2l) are particle-particle-hole-hole terms. Note that only particle-hole terms are contained in conventional RPA correlation methods, see table 2 . The additional terms $\left(2 \mathrm{~b}^{\mathrm{lx}}\right),\left(2 \mathrm{~d}^{\mathrm{lx}}\right),\left(2 \mathrm{~g}^{\mathrm{lx}}\right)$ and $\left(2 \mathrm{~h}^{\mathrm{lx}}\right)$ originate from local exchange and occur only in RPA methods based on EXX. Note that the terms (2d) and (2g) as well as (2e) and (2f) are identical if real-valued orbitals are used.

term spin-orbital expression

(1a) $\quad \frac{1}{2} \operatorname{Tr}[\overline{\mathbf{C C}}]$

(1b) $\frac{1}{2} \operatorname{Tr}[\overline{\mathbf{C K}}]$

(2a) $\operatorname{Tr}[\overline{\mathbf{C}} \mathbf{C} \overline{\mathbf{C}}]$

(2b) $-\operatorname{Tr}[\overline{\mathbf{C}} \overline{\mathbf{C}}]$

(2c) $\operatorname{Tr}[\overline{\mathbf{K}} \mathbf{C} \overline{\mathbf{K}}]$

(2d) $\operatorname{Tr}[\overline{\mathbf{K}} \mathbf{J} \overline{\mathbf{C}}]$

(2e) $-\operatorname{Tr}[\overline{\mathbf{K}} \mathbf{C \mathbf { C }}]$

(2f) $-\operatorname{Tr}[\overline{\mathbf{C}} \mathbf{C} \overline{\mathbf{K}}]$

(2g) $\quad \operatorname{Tr}[\overline{\mathbf{C}} \mathbf{J} \overline{\mathbf{K}}]$

(2h) $-\operatorname{Tr}[\overline{\mathbf{K}} \mathbf{J} \overline{\mathbf{K}}]$

$\left(2 \mathrm{~b}^{\mathrm{lx}}\right) \quad-\operatorname{Tr}[\overline{\mathbf{C}} \boldsymbol{\Delta} \overline{\mathbf{C}}]$

$\left(2 \mathrm{~d}^{\mathrm{lx}}\right) \quad \operatorname{Tr}[\overline{\mathbf{C}} \boldsymbol{\Delta} \overline{\mathbf{K}}]$

$\left(2 \mathrm{~g}^{\mathrm{lx}}\right) \quad \operatorname{Tr}[\overline{\mathbf{K}} \boldsymbol{\Delta} \overline{\mathbf{C}}]$

$\left(2 \mathrm{~h}^{\mathrm{lx}}\right) \quad-\operatorname{Tr}[\overline{\mathbf{K}} \boldsymbol{\Delta} \overline{\mathbf{K}}]$

(2i) $\quad \frac{1}{2} \sum_{i j, a b c d} \bar{C}_{i a, j d} E_{a d}^{c b} \bar{C}_{i c, j b}$

(2j) $\quad \frac{1}{2} \sum_{i j k l, a b} \bar{C}_{i a, l b} S_{i l}^{j k} \bar{C}_{j a, k b}$

$(2 \mathrm{k})$

$-\frac{1}{2} \sum_{i j k l, a b} \bar{C}_{i a, l b} S_{i l}^{j k} \bar{K}_{j a, k b}$

(2l) $\quad-\frac{1}{2} \sum_{i j, a b c d} \bar{C}_{i a, j d} E_{a d}^{c b} \bar{K}_{i c, j b}$ 


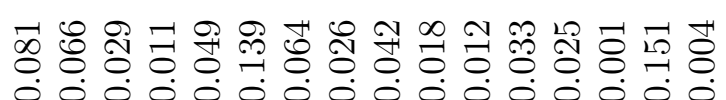
i $\begin{gathered}0 \\ 0\end{gathered}$

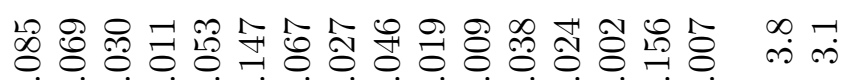

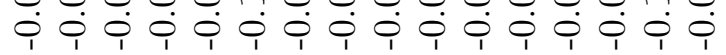

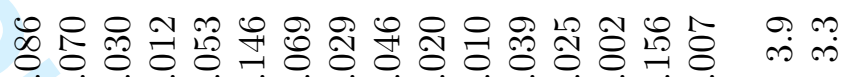

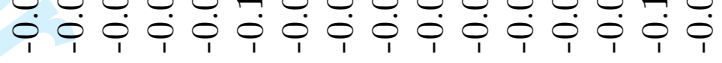

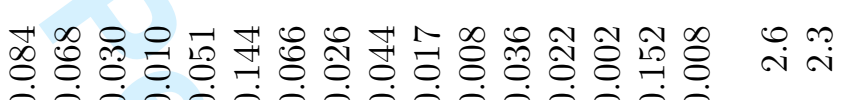
0
1

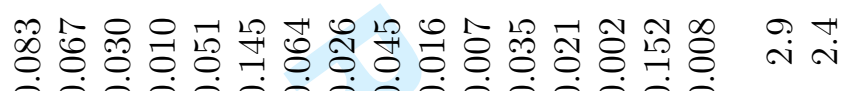

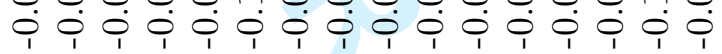

ஜ

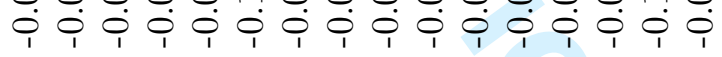

F

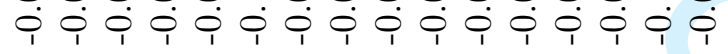

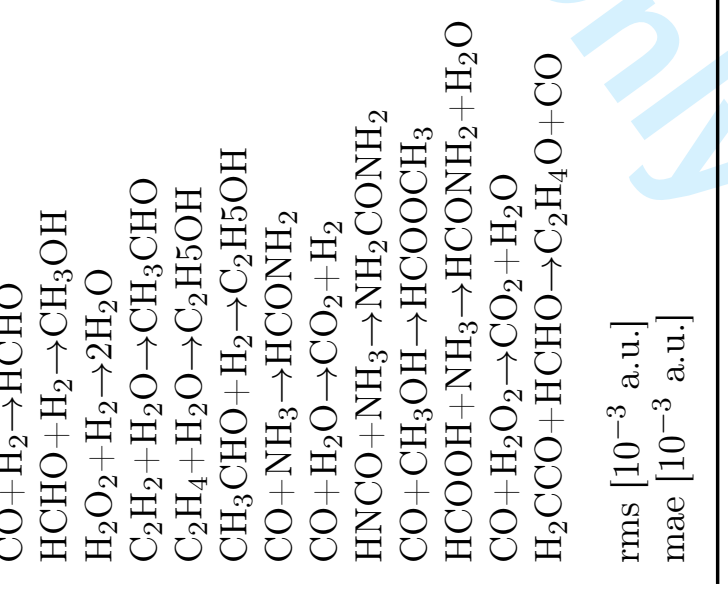




\section{Page 43 of $45 \quad$ Molecular Physics}

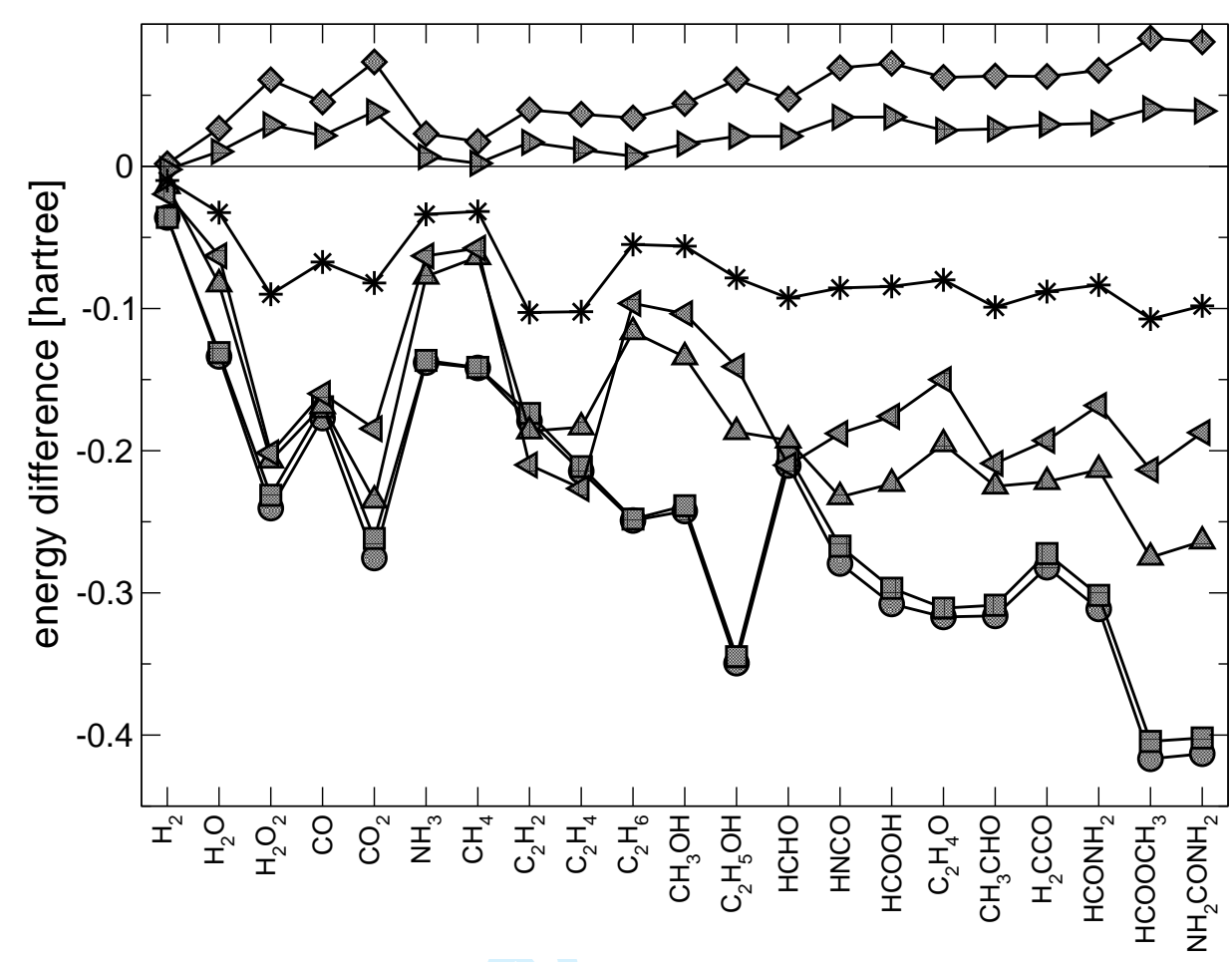

-0 PBE-dRPA EXX-dRPA $\triangle$ EXX-SOSEX $\triangle \triangle E X X-N R P A 1$ $4-4$ EXX-NRPA2 $\rightarrow$ EXX-NRPA3 * $*$ EXX-NRPA4

Figure 1. Energy differences to $\operatorname{CCSD}(\mathrm{T})$ reference energies for various Kohn-Sham orbital based RPA methods.

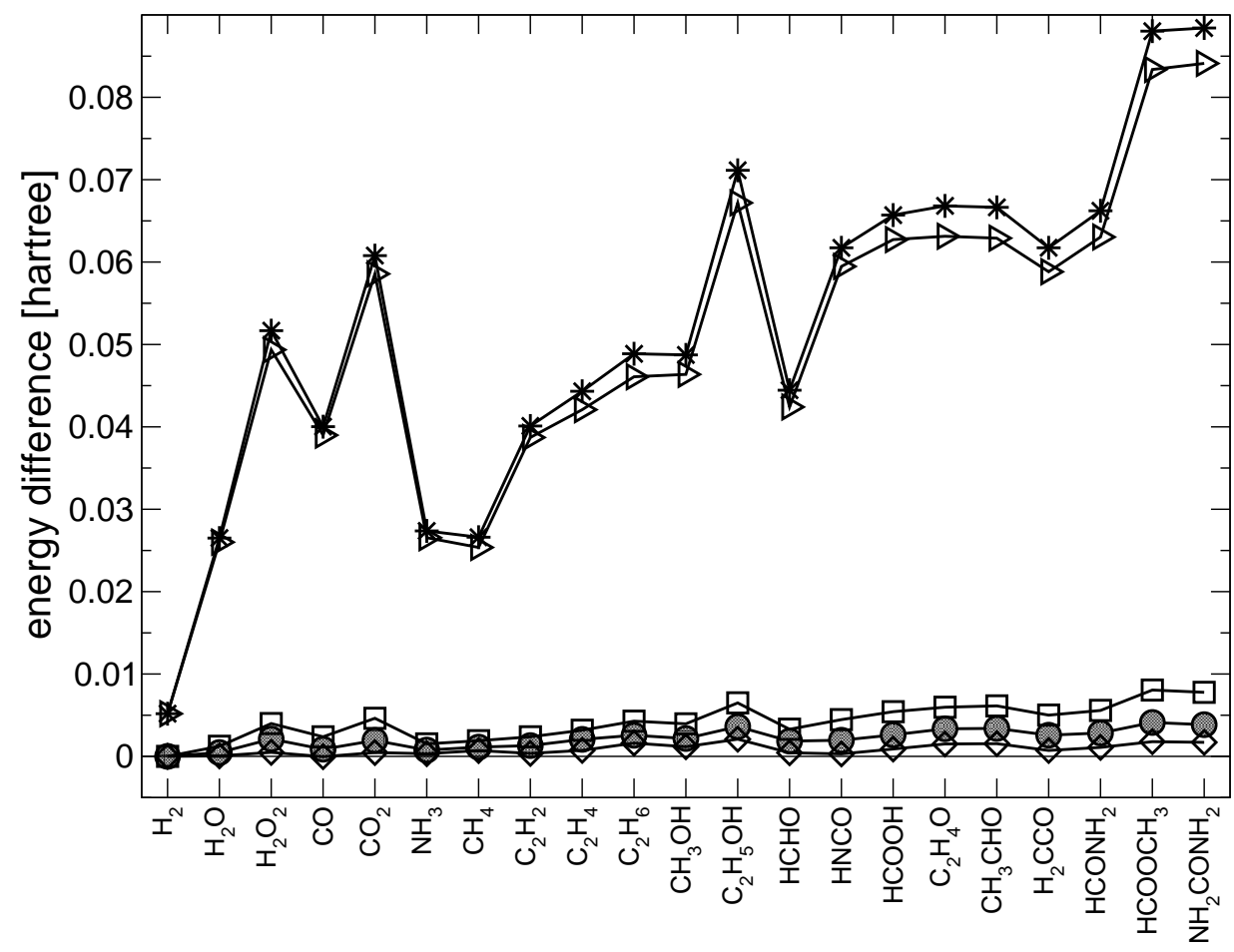

- EXX-NRPA3 $\square \boxminus$ EXX-RPA $\diamond \diamond \mathrm{EXX}-\mathrm{RPA}[\infty]$ $\leftrightarrow A C-R P A$ * * AC-RPA(EXX)

Figure 2. Energy differences to NRPA3 energies(Eq. (69)) for the methods EXX-NRPA3 (Eq. (107)), EXX-RPA, EXX-RPA[ $\infty$ ] (Eq. (100)), AC-RPA (Eq. (76)) and AC-RPA(EXX). 
Taylor \& Francis and I.T. Consultant
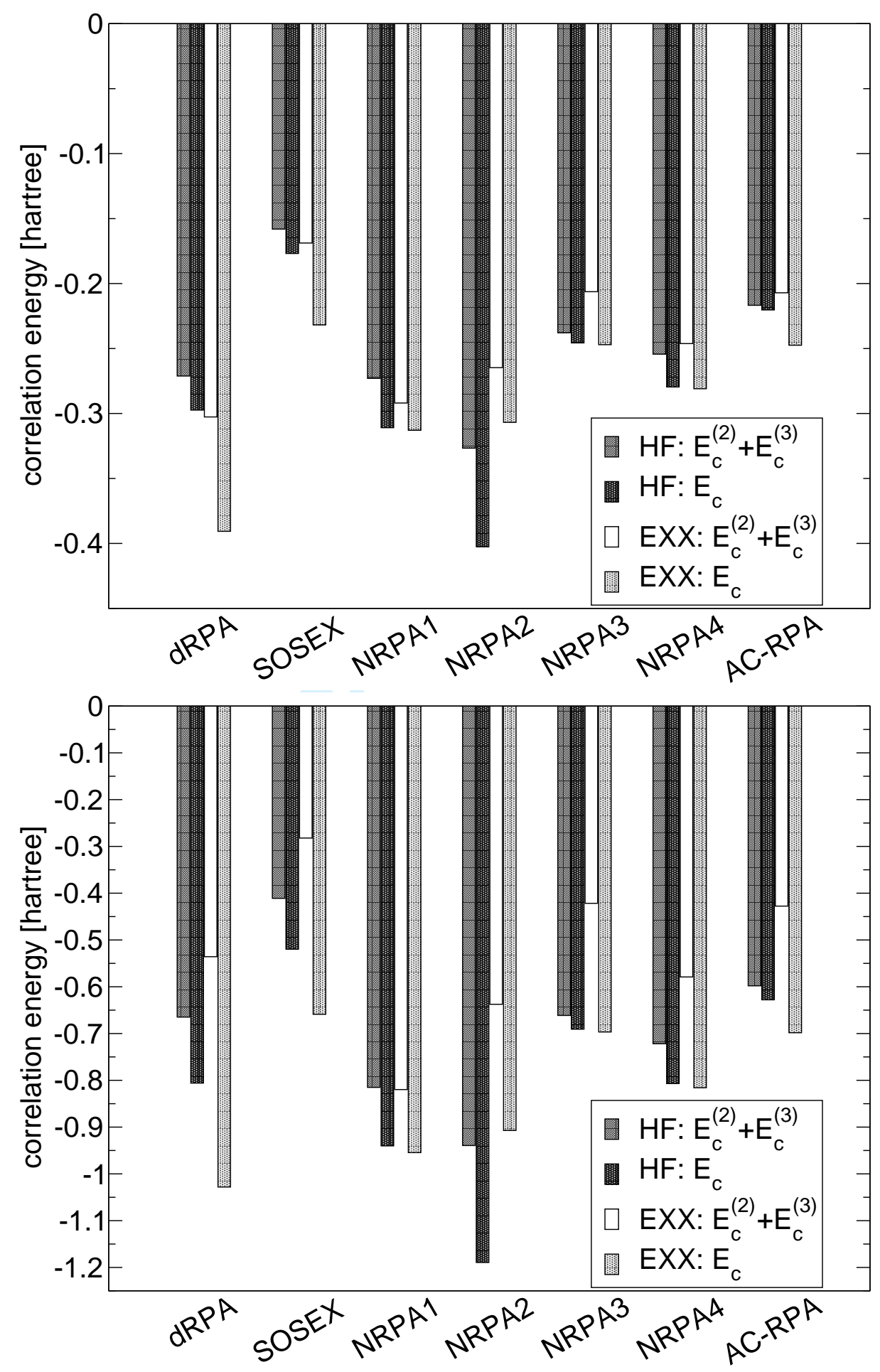

Figure 3. Correlation energies for various RPA methods of the $\mathrm{CH}_{4}$ (top diagram) and $\mathrm{HCOOH}$ molecule (bottom diagram). The first and third bars show the sum of the second- and third-order energy for the HF-based and EXX-based RPA methods and the second and fourth bars show the total correlation energies for the HF-based and EXX-based RPA methods, respectively. 

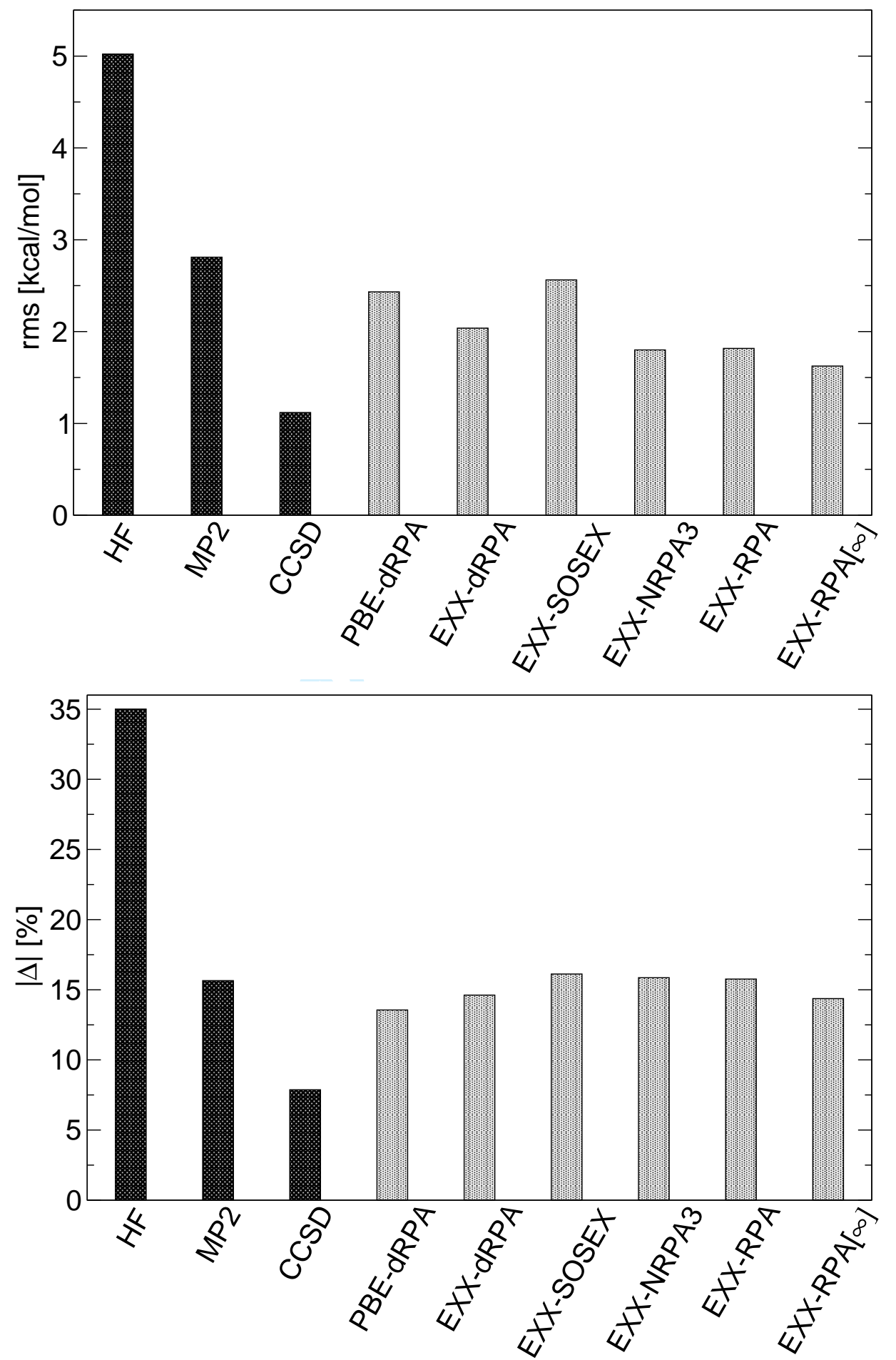

Figure 4. Root mean squared errors (rms) and relative percentual deviations for Hartree-Fock and various correlation methods to $\operatorname{CCSD}(\mathrm{T})$ reaction energies shown in table 3 . 